\title{
Basics of Generalized Unitarity
}

\author{
Zvi Bern and Yu-tin Huang \\ Department of Physics, UCLA, Los Angeles, CA 94305-4060, USA \\ E-mail: bern@physics.ucla.edu, yhuang@physics.ucla.edu
}

\begin{abstract}
We review generalized unitarity as a means for obtaining loop amplitudes from on-shell tree amplitudes. The method is generally applicable to both supersymmetric and non-supersymmetric amplitudes, including non-planar contributions. Here we focus mainly on $\mathcal{N}=4$ Yang-Mills theory, in the context of onshell superspaces. Given the need for regularization at loop level, we also review a sixdimensional helicity-based superspace formalism and its application to dimensional and massive regularizations. An important feature of the unitarity method is that it offers a means for carrying over any identified tree-level property of on-shell amplitudes to loop level, though sometimes in a modified form. We illustrate this with examples of dual conformal symmetry and a recently discovered duality between color and kinematics.

This article is an invited review for a special issue of Journal of Physics A devoted to "Scattering Amplitudes in Gauge Theories".
\end{abstract}




\section{Introduction}

The unitarity method [1, 2, 3, 4, 15, 6, 7, 8, 9, 10, 11, 12, is at present the most powerful general means for obtaining loop-level scattering amplitudes. It applies to any massless non-supersymmetric or supersymmetric theory, including their non-planar contributions. Here we will summarize basic ideas for applying generalized unitarity as a means for obtaining loop amplitudes from on-shell tree amplitudes. We will focus on $\mathcal{N}=4$ super-Yang-Mills theory, in particular explaining how sums over intermediate states are conveniently evaluated using on-shell superspaces. Up-to-date discussions of applications and further details of generalized unitarity may be found in the chapters of this review by Britto [13, by Carrasco and Johansson [14] and by Ita [15].

The unitarity method has been applied to a wide variety of problems in phenomenology and more theoretical aspects of quantum field theory. This includes state-of-the-art predictions of LHC physics (for example, see refs. [9, 16, 17]), including the first next-to-leading-order QCD calculation with five final state objects (including jets) [17]. On the more theoretical side, it has been used to open a new venue [18] for studying Maldacena's AdS/CFT correspondence [19] and perhaps eventually leading to a solution of planar $\mathcal{N}=4$ super-Yang-Mills theory. It has also been used to unravel a dual conformal invariance in planar loop amplitudes of $\mathcal{N}=4$ super-YangMills theory [20, 21, 22, 23, 24, 25, 12, 26, 27, 28, 29]. Applications to supergravity theories [30, 10, 31] have led to results that have rejuvenated hope for the absence of ultraviolet divergences for $\mathcal{N}=8$ supergravity. (For recent discussions of this issue see refs. [32, 33, 34.) More recently, it has also played a key role in the conjecture of an all-loop-order duality between color and kinematics and the associated double-copy structure of gravity amplitudes in terms of gauge theory ones [35, 36]. Very recently it has also played a role in a new means for recursively finding multiloop multileg integrands in planar $\mathcal{N}=4$ super-Yang-Mills theory [37, 38].

The key feature of the unitarity method is that it constructs loop amplitudes directly from on-shell tree amplitudes. This makes it possible to carry over any newly identified property or symmetry of tree-level amplitudes to loop level. This may be contrasted with Feynman diagrammatic methods, whose diagrams are inherently gauge dependent and off shell in intermediate states, making it difficult to exploit any simplifying properties of the on-shell tree amplitudes.

Unitarity has a long history in quantum field theory since its inception. For a discussion of applications of unitarity during the 1960's, see ref. [39]. However, a variety of difficulties prevented its widespread use as a means of constructing amplitudes, especially after the rise of gauge theories in the 1970's. These difficulties included

the need for subtractions (meaning undetermined pieces), the inability to construct amplitudes beyond four points and difficulties with massless particles. These basic difficulties were overcome with the advent of the modern unitarity method [1].

Over the years there have been a number of important refinements to the unitarity method. Generalized unitarity [39] (where multiple internal lines are placed on shell, 
subdividing a loop amplitude into more than two pieces) was first applied in ref. [4] as a means for simplifying loop calculations. An important more recent development is the use of complex momenta [40] by Britto, Cachazo and Feng [7], leading to the realization that at one-loop in four dimensions, quadruple cuts directly determine the coefficients of all box integrals by freezing the loop integration. Powerful new methods for dealing with triangle and bubble integrals at one-loop, as well as rational terms have also been developed [9, 41]. These are described in other chapters of this review [13, 15]. At higher loops, efficient means of constructing the integrands of amplitudes, including non-planar contributions, have also been devised [8, 10, 11, 37], as discussed in some detail in another chapter of this review [14].

Although the unitarity method applies just as well to supersymmetric and nonsupersymmetric theories, in general it is simpler to deal with the supersymmetric case because of the simpler analytic structure of such amplitudes. In particular, it turns out that the better power counting of supersymmetric theories allows all terms in oneloop scattering amplitudes to be readily captured by cuts composed of four-dimensional massless tree amplitudes. Indeed, the first applications of the unitarity method were for one-loop supersymmetric amplitudes with arbitrary numbers of external legs, leading to remarkably compact results [1]. For QCD or even supersymmetric theories at higher loops, the situation is more complex. The source of this additional complexity is that contributions that formally vanish in four dimensions may actually contribute because they interfere with divergences. At one-loop on-shell recursion relations can be used to construct the rational terms, entirely in four dimensions [41. More generally, by computing in $D=4-2 \epsilon$ dimensions all rational terms are automatically captured [2]. A convenient way to implement this, is by using six-dimensional helicity [42, 43, 44]. This offers many of the advantages of four-dimensional helicity, but meshes well with regularization of the divergences, in a manner consistent with unitarity [12]. Besides regularization issues, there are a number of interesting questions in higher-dimensional theories accessible via six-dimensional techniques. For example, it has recently been used to confirm [12] that four-dimensional cuts do capture all terms in the four-loop four-point amplitudes of $\mathcal{N}=4$ super-Yang-Mills theory [11] and $\mathcal{N}=8$ supergravity [31], which are relevant for higher-dimensional studies of ultraviolet divergences in these theories. Another interesting application would be to confirm or refute the very interesting recent proposal that maximally supersymmetric Yang-Mills theory may be ultraviolet finite in five dimensions [45].

The present chapter of this review is organized as follows. After explaining the basics of the unitarity method, we illustrate this method for the one-loop four-point amplitude of $\mathcal{N}=4$ super-Yang-Mills. We start by working out the amplitude in components. Then we turn to more powerful superspace formalisms, first in four dimensions and then in six dimensions. We then present two nontrivial examples of how the unitarity method allows us to carry over on-shell tree-level properties to loop level. In the first of these examples we show that dual conformal covariance of tree amplitudes implies that loop-level integrands must transform nicely as well. In the second we will 
describe a duality between color and kinematics, showing the transition from trees to loops. In general, tree properties carry over straightforwardly to generalized cuts that decompose a loop amplitude into a product of tree amplitudes. However, as the second example illustrates it can be nontrivial to demonstrate that a given property holds for the complete loop amplitude.

\section{Basics of the unitarity method}

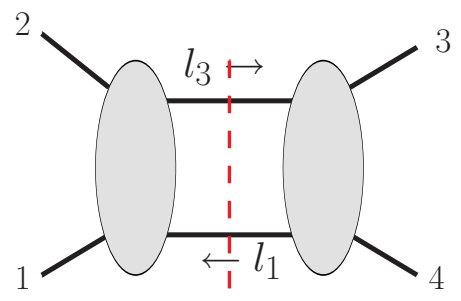

(a)

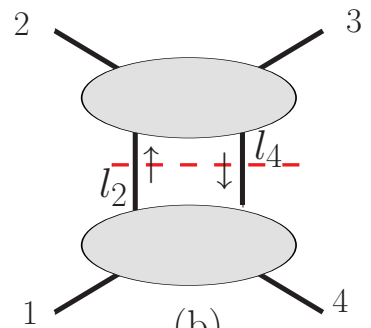

(b)

Figure 1. The $s$ and $t$ channel two-particle cuts of the one-loop four-point amplitude.

\subsection{Overview}

The simplest unitarity cuts to consider are the two-particle cuts. These are obtained by putting two intermediate lines on shell, as illustrated in figure 1 for a four-point one-loop amplitude. For example, the $s$ channel cut in figure 1(a) is given by

$$
C_{s}=\sum_{\text {states }} A^{\text {tree }}\left(-l_{1}, 1,2, l_{3}\right) A^{\text {tree }}\left(-l_{3}, 3,4, l_{1}\right),
$$

where the sum runs over all physical states in the theory. The cuts are evaluated using momenta that place all intermediate cut momenta on shell, $l_{i}^{2}=m_{i}^{2}$, where the $m_{i}$ are masses. Here we will take the theory to be massless. Cuts are usually taken as including phase-space integrals, but for our purposes it is simpler to define them as not including the phase-space integration.

An especially useful class of cuts are those that decompose a loop amplitude into a sum over $m$ tree amplitudes of form,

$$
C=\sum_{\text {states }} A_{(1)}^{\text {tree }} A_{(2)}^{\text {tree }} A_{(3)}^{\text {tree }} \cdots A_{(m)}^{\text {tree }}
$$

where the sum runs over all physical states that can cross the cuts. In $\mathcal{N}=4$ superYang-Mills theory, it is especially useful to consider the maximal cuts [8], (also referred to as "leading singularities" [46]), where the maximum number of propagator lines are placed on shell. Another useful class are single cuts where only a single internal line is placed on shell [47]; these have played an important role in the construction of planar integrands of $\mathcal{N}=4$ super-Yang-Mills theory via on-shell recursion [37]. 
In general, the complete amplitude is determined from a "spanning set" of cuts. Such sets are found by considering all potential independent contributions to the integrand that can enter an amplitude (and which do not integrate to zero), based on power counting or other constraints. One simply needs to ensure that all terms are non-vanishing in at least one cut that can then be used to determine its coefficient. In the $\mathcal{N}=4$ case one can often construct an ansatz for the entire amplitude using various conjectured properties. Once one has an ansatz, by confirming it over the spanning set, either numerically or analytically, we have a proof of the correctness of the ansatz.

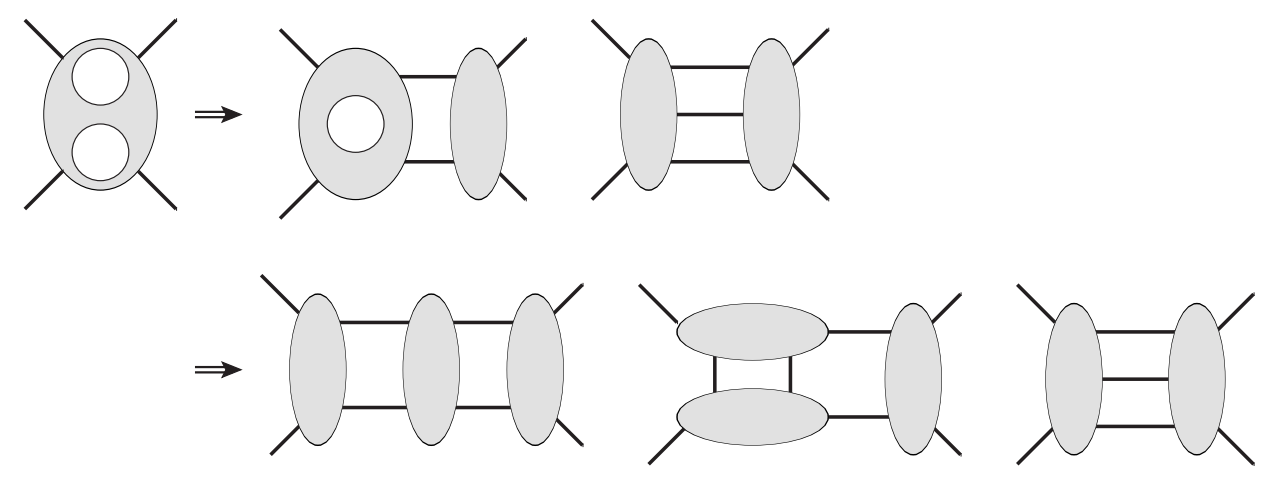

Figure 2. The procedure for obtaining a spanning set of cuts for a planar twoloop four-point amplitude. Only topologically distinct diagrams are shown. The exposed intermediate lines are all placed on shell.

One simple spanning set is obtained from the set of standard unitarity cuts, where a given amplitude is split into two lower-loop amplitudes, each with four or more external legs. At $L$ loops, this is given by all cuts starting from the two-particle cut to the $(L+1)$-particle cut in all channels. We can convert this to a spanning set involving only tree amplitudes by iterating this process until no loops remain. To illustrate this procedure, consider the two-loop four-point amplitude displayed in figure 2. In the first stage this is decomposed using two- and three-particle cuts. Then in the second stage, the one-loop amplitude appearing in the two-particle cut is further decomposed into its two-particle cuts. In this figure we display only the topologically distinct diagrams; the complete decomposition is determined by considering all cuts with distinct labels. If the amplitudes are color ordered then we need to maintain a fixed ordering of legs, depending on which planar or non-planar contribution is under consideration. On the other hand, if they are dressed with color then the distinct permutations of legs enter each cut automatically.

Another spanning set that is especially useful in $\mathcal{N}=4$ super-Yang-Mills theory is obtained [8, 10, 11] by starting from "maximal cuts", where the maximum numbers of internal propagators are placed on shell. These maximal cuts decompose the amplitudes into products of three-point tree amplitudes, summed over the states crossing the cuts. To construct a spanning set we systematically release cut conditions one by one, first considering cases with one internal line off shell, then two internal lines off shell and so 
forth. Each time a cut condition is released, potential contact terms which would not be visible at earlier steps are captured. The process terminates when the only remaining potential contact terms exceed power counting requirements of the theory (or integrate to zero in dimensional regularization). For the case of maximal supersymmetryespecially in the planar case - there are a large variety of additional tricks and techniques for obtaining contributions efficiently [8, 46, 11, 37, 38. Many of these are discussed further by Carrasco and Johansson in another chapter of this review [14].

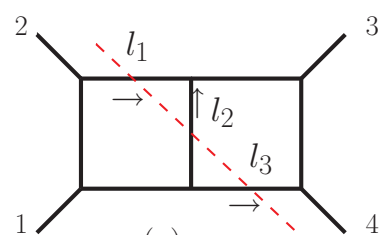

(a)

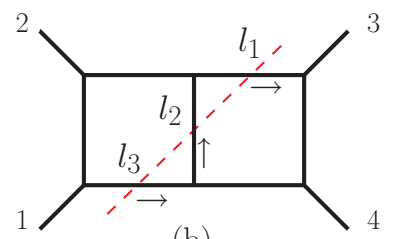

(b)

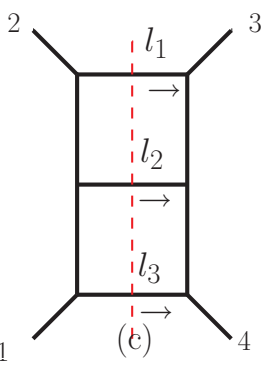

Figure 3. The double-box three-particle cuts. The cut contributions (a) and (b) are two distinct cut contributions of the same horizontal double-box integral. The vertical double box has only a single contribution to the three-particle cut.
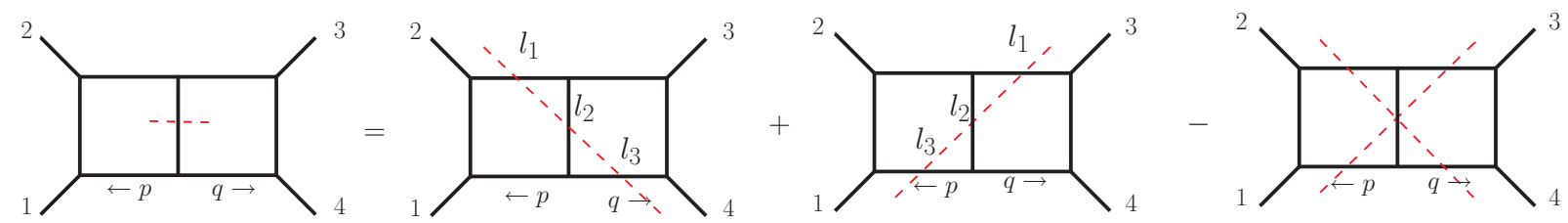

Figure 4. An example showing how cuts are combined to obtain contributions with fewer cut conditions. In this equation the momentum labels of the different cut contributions need to be aligned, before being combined. On the right hand side, the only remaining cut conditions

Given a spanning set of unitarity cuts, the task is to then find an expression for the integrand of the amplitude with the correct cuts in all channels. This can be done either in a forward or reverse direction. In the forward direction the different cuts are merged into integrands with no cut conditions using a merging procedure described in ref. [6]. In the reverse direction we first construct an ansatz for the amplitude containing unknown parameters which are then determined by taking generalized cuts of the ansatz and comparing to the cuts of the amplitude. The reverse direction is usually preferred because we can expose desired properties, simply by imposing them on the ansatz and then checking if its unitarity cuts are correct.

\subsection{Cut merging procedure}

To illustrate the forward way of proceeding, consider a two-loop four-point amplitude. In particular, consider those contributions which contain the propagators of the double- 
box integral displayed in figure 3 ,

$$
\int \frac{d^{D} p}{(2 \pi)^{D}} \frac{d^{D} q}{(2 \pi)^{D}} \frac{N}{p^{2}\left(p-k_{1}\right)^{2}\left(p-k_{1}-k_{2}\right)^{2}(p+q)^{2} q^{2}\left(q-k_{4}\right)^{2}\left(q-k_{3}-k_{4}\right)^{2}} .
$$

The key task is to determine the numerator factor $N$ from the unitarity cuts. As illustrated in figure 3 , the three-particle cut in the $s_{12}$ channel will have three contributions. The horizontal double box gives two distinct contributions to the cut, while the vertical double box gives only a single contribution. When reassembling the contribution from the cuts we need to account for this relative double count in the horizontal box. A procedure for doing so is by adding together the two horizontal box contributions, but subtracting out the overlap, to prevent a double count, as illustrated in figure 4 .

Prior to adding the two contributions (a) and (b) in figure 3 we must switch from cut labels used in diagram (a) to the diagram momentum labels, $p$ and $q$, so that uniform momentum labels are used when combining the contributions. Note that the contributions (a) and (b) each require a different change of variables. For diagram (a) we require

$$
l_{1}=p-k_{1}-k_{2}, \quad l_{2}=-p-q, \quad l_{3}=q,
$$

while for diagram (b) we require

$$
l_{1}=-q+k_{3}+k_{4}, \quad l_{2}=p+q . \quad l_{3}=-p,
$$

The third term on the right hand side of figure 4 is the "overlap" between the numerator contributions (a) and (b) which must be subtracted out to prevent double counts. The overlap term is determined by taking contribution (a) and imposing the additional cut conditions of diagram (b). In carrying this out, it is important that terms which vanish by the cut conditions are set to zero. This is best accomplished by expressing the numerator in terms of inverse propagators, whenever possible. A key consistency condition is that the overlap terms need to be the same if we swap the roles of (a) and (b). (In explicit calculations it is helpful to always check this consistency condition, since it will catch most errors.)

This merging procedure generalizes to any set of cuts. In general, we can write a gauge-theory amplitude as a sum over integrals,

$$
\mathcal{A}_{n}^{(L)}=g^{2 L+n-2} \sum_{\text {diags. } i} \frac{1}{S_{i}} c_{i} I_{i},
$$

where the $S_{i}$ are symmetry factors, the $c_{i}$ color factors, the $I_{i}$ some loop integrals and the sum runs over all contributing integrals. The loop integrals are of the form,

$$
I_{i}=\int \prod_{j=1}^{L} \frac{d^{D} l_{i}}{(2 \pi)^{D}} \frac{n_{i}}{\prod_{j \in \alpha_{i}} p_{j}^{2}}
$$

where the $n_{i}$ are numerators. The numerators can be local or non-local depending on the chosen organization. The product in the denominator runs over all propagators in the graph. Each cut contribution is then assigned to a graph according to the propagators 
it contains and the merging procedure is carried out for each graph. For each set of propagators specifying an integral function, one must add together the contributions from each cut, subtracting any overlap. A detailed discussion of this procedure may be found in ref. [6].

We note that the merging procedure is not limited to planar supersymmetric theories and has been successfully applied to QCD [6]. In special cases, such as $\mathcal{N}=4$ super-Yang-Mills theory at one loop, the merging procedure is rather simple, because we can make use of the fact that the amplitude is determined solely from box integrals [1], whose coefficients may be determined directly from quadruple cuts [7].

\subsection{Merging cuts via an ansatz}

In practical calculations, it is usually simpler to work in the reverse direction, by first constructing an ansatz for the numerators containing all potential terms that can appear. The numerators can depend on the momenta, polarizations and spinors and contain parameters to be determined from the cuts. When using superamplitudes, the numerators will depend on anticommuting parameters as well.

To determine the unknown numerical coefficients we take generalized cuts of the ansatz and systematically set these equal to the cuts of the loop amplitude. Usually it is best to enforce a set of auxiliary constraints, such as demanding an upper limit to the number of powers of loop momenta that that can appear in the numerators of the integrands. Depending on the situation, one can, for example, impose that each numerator is a polynomial in the loop and external momenta. One can greatly reduce the number of cuts required to determine the amplitude by feeding in additional information, such as dual conformal symmetry for the planar $\mathcal{N}=4$ super-Yang-Mills amplitudes [8, 38]. This procedure is simplest for the four-point amplitudes of $\mathcal{N}=4$ super-Yang-Mills theory, where it turns out that the ratio between the loop integrand and the tree amplitudes is a rational function solely of Lorentz invariant scalar products, at least through four loops and very likely beyond this [48, 22, 8, 11]. For higher-point amplitudes, similar ratios can contain either spinor products or Levi-Civita tensors. If it turns out that no solution to the cut conditions is found, the ansatz then needs to be enlarged by removing auxiliary constraints.

A given cut configuration will not completely fix the ansatz as the numerator may be freely modified by adding terms that vanish on the cut under study. Consider, for example, a particular two-particle cut with cut momenta labeled $l_{1}$ and $l_{2}$. Expressions proportional to $l_{1}^{2}=0$ and $l_{2}^{2}=0$ are not constrained by this particular cut. Such terms are instead constrained by other cuts. After information from all cuts is included, the only remaining ambiguities are terms that are free of cuts in every channel. In the full amplitude these ambiguities add up to zero, representing the freedom to reexpress the amplitude in different equivalent forms. This freedom allows us to find representations with different desirable properties, such as manifest symmetries or power countings [10, 36, 11]. 
For multiloop calculations in $\mathcal{N}=4$ super-Yang-Mills theory, it is usually helpful to organize the evaluation of the cuts following the method of maximal cuts [8]. This allows us to focus on a relatively small number of terms, as well as reducing the complexity of each cut. A related procedure, valid for maximally supersymmetric amplitudes, makes use of "leading-singularities" 46]. These leading singularities, which include additional hidden singularities, likely determine any maximally supersymmetric amplitude, once additional symmetry information is incorporated [49, 38].

Similar constructions can also be done in supergravity theories. However, it is generally simpler to construct loop amplitudes in these theories by first evaluating corresponding super-Yang-Mills loop amplitudes which can then be using directly in the evaluation of the supergravity cuts [50, 30, 31], using either the tree-level KLT [51] relations or the double-copy BCJ relations [35]. In fact, it appears likely that one can read off multiloop supergravity amplitudes directly from the corresponding gauge-theory amplitudes after they are arranged in a form that satisfies a color-kinematics duality [36].

\subsection{Regularization issues}

One important technical issue is that amplitudes are in general divergent and need to be regularized. For most purposes, the most convenient regularization scheme is dimensional regularization. For planar $\mathcal{N}=4$ super-Yang-Mills theory only infrared divergences are present, and in this case a massive Higgs regulator has proven to be especially convenient [52. Interestingly, this form of massive regularization is closely related to dimensional regularization, and obtained by treating the extra dimensional components of momenta as masses not to be integrated over [12].

At one loop, all contributions to the amplitudes of massless supersymmetric theories are determined completely by their four-dimensional cuts [1]. Unfortunately, no such theorem has been proven at higher loops. There is, however, substantial evidence that it holds for four-point amplitudes in this theory through six loops [22, 30, 8, 11, 12]. We do not expect that it will continue for higher-point amplitudes. Indeed, we know that for two-loop six-point amplitudes, terms which vanish in $D=4$ do contribute in dimensional regularization [53]. However, it may well turn out that all terms not determined by four-dimensional massless cuts have a predictable universal structure, at least near four dimensions. Interestingly, experience indicates that when using the massive regulator, we can safely drop all terms of order $m$, where $m$ is the effective mass obtained from the extra-dimensional components [52, 37, 54].

Until theorems are proven determining when four-dimensional massless cuts are sufficient to guarantee that all terms are captured, by evaluating cuts in higher dimensions we can ensure their reliability. In general, using $D$-dimensional momenta [2, 3] makes calculations significantly more clumsy, because powerful spinor and superspace methods [55] are no longer applicable. However, thanks to refs. [42, 44], we now have helicity and superspace formalisms in six dimensions analogous to the well known four-dimensional ones. In many cases this is sufficient, allowing for straightforward 
constructions of dimensionally or massively regularized amplitudes [12]. This then offers a convenient way to construct regularized integrands that are guaranteed to be consistent with unitarity.

\section{Sample Calculation: One-loop four-point amplitude in maximal super-Yang-Mills theory}

For relatively simple amplitudes, the sum over states in a cut (2) can be evaluated straightforwardly in components [50]. As we discuss in subsequent sections, for more complex situations where the bookkeeping becomes more difficult, the sums over states are best handled [56, 57, 58, 12, 59, 60] using on-shell superspaces [61, 43, 44].

As a simple example where a component analysis is adequate, consider a fourgluon amplitude of $\mathcal{N}=4$ super-Yang-Mills theory. This amplitude was first computed in ref. [62], as the low-energy limit of a type I string. For the purposes of illustrating the cut construction, we may view this amplitude as an ordinary gauge-theory amplitude but with a particular matter content: one gluon, four gluinos and six real scalars all in the adjoint representation. In supersymmetric theories amplitudes with identical helicity or with one leg of opposite helicity (in an all outgoing convention) vanish to all loop orders by a supersymmetry identity 63]. (A discussion of supersymmetry identities can be found in another chapter of this review [34.) The first non-trivial case to consider is the four-point case with two negative and two positive helicities, $A_{4}^{\mathcal{N}=4}\left(1^{-}, 2^{-}, 3^{+}, 4^{+}\right)$.

In general, we can write the color-ordered amplitude as

$$
A_{4}^{\mathcal{N}=4}\left(1^{-}, 2^{-}, 3^{+}, 4^{+}\right)=-i A_{4}^{\text {tree }} \int \frac{d^{4-2 \epsilon} p}{(2 \pi)^{4-2 \epsilon}} \frac{N}{p^{2}\left(p-k_{1}\right)^{2}\left(p-k_{1}-k_{2}\right)^{2}\left(p+k_{4}\right)^{2}},
$$

where we have extracted an overall factor of the tree amplitude. (The amplitude is the coefficient of the color trace $N_{c} \operatorname{tr}\left(T^{a_{1}} T^{a_{2}} T^{a_{3}} T^{a_{4}}\right)$ for an $\mathrm{SU}\left(N_{c}\right)$ gauge group.) Our task is to determine the numerator factor $N$ from the cuts. We have chosen to write the amplitude entirely as a box integral. In this representation, if any triangle or bubble contributions were to appear they would enter as inverse propagators in the numerator canceling propagators.

For simplicity, we will work this out using four-dimensional momenta. As mentioned above, in general, this can lead to terms being dropped, but supersymmetric amplitudes at one loop are known not to contain additional terms [1]. In carrying out this calculation we assume the relevant tree-level amplitudes are already known, so we just quote their form [64]. First consider the $s$-channel cut depicted in figure 1(a). The necessary tree amplitudes are the four-gluon amplitudes,

$$
\begin{aligned}
& A_{4}^{\text {tree }}\left(-l_{1}^{+}, 1^{-}, 2^{-}, l_{3}^{+}\right)=i \frac{\langle 12\rangle^{4}}{\left\langle-l_{1} 1\right\rangle\langle 12\rangle\left\langle 2 l_{3}\right\rangle\left\langle l_{3}-l_{1}\right\rangle}, \\
& A_{4}^{\text {tree }}\left(-l_{3}^{-}, 3^{+}, 4^{+}, l_{1}^{-}\right)=i \frac{\left\langle-l_{1} l_{3}\right\rangle^{4}}{\left\langle-l_{3} 3\right\rangle\langle 34\rangle\left\langle 4 l_{1}\right\rangle\left\langle l_{1}-l_{3}\right\rangle},
\end{aligned}
$$

where $\langle i j\rangle$ denote spinor inner products following the notation of ref. [65]. All other combinations of helicities of the intermediate lines cause at least one of the tree 
amplitudes on either side of the cut to vanish. (The outgoing-particle helicity convention means that the helicity label for each intermediate line flips when crossing the cut.) Remarkably, for this cut only the gluon loop contributes; for fermion or scalar loops at least one of the two tree amplitudes vanish. Using the tree amplitudes (9), the cut in the $s$ channel depicted in figure 1(a) is simply,

$$
C_{s}=\frac{i\langle 12\rangle^{4}}{\left\langle l_{1} 1\right\rangle\langle 12\rangle\left\langle 2 l_{3}\right\rangle\left\langle l_{3} l_{1}\right\rangle} \frac{i\left\langle l_{1} l_{3}\right\rangle^{4}}{\left\langle l_{3} 3\right\rangle\langle 34\rangle\left\langle 4 l_{1}\right\rangle\left\langle l_{1} l_{3}\right\rangle},
$$

where we used the assignment that $|-p\rangle=-|p\rangle$ and $\mid-p]=\mid p]$ such that $|-p\rangle[-p \mid=-p$. To put this into a form more reminiscent of integrals encountered in Feynman diagram calculations we rationalize the denominators using, for example,

$$
\frac{1}{\left\langle 2 l_{3}\right\rangle}=-\frac{\left[2 l_{3}\right]}{\left(p-k_{1}\right)^{2}}
$$

where we set $l_{1}=p, l_{3}=p-k_{1}-k_{2}$ and we used the on-shell conditions $l_{1}^{2}=l_{3}^{2}=0$. Performing these simplifications yields

$$
C_{s}=i A_{4}^{\text {tree }} \frac{\tilde{N}}{\left(p-k_{1}\right)^{4}\left(p+k_{4}\right)^{4}},
$$

where we have extracted a overall factor of the tree amplitude from the amplitude,

$$
A_{4}^{\text {tree }}\left(1^{-}, 2^{-}, 3^{+}, 4^{+}\right)=i \frac{\langle 12\rangle^{4}}{\langle 12\rangle\langle 23\rangle\langle 34\rangle\langle 41\rangle} .
$$

The numerator $\tilde{N}$ is given by

$$
\begin{aligned}
\tilde{N} & =\left[l_{1} 1\right]\langle 14\rangle\left[4 l_{1}\right]\left\langle l_{1} l_{3}\right\rangle\left[l_{3} 3\right]\langle 32\rangle\left[2 l_{3}\right]\left\langle l_{3} l_{1}\right\rangle \\
& =\operatorname{tr}_{+}\left[l_{1} 14 l_{1} l_{3} 32 l_{3}\right] \\
& =-4 \operatorname{tr}_{+}[4321] l_{1} \cdot k_{1} l_{1} \cdot k_{4}=-s t\left(p-k_{1}\right)^{2}\left(p+k_{4}\right)^{2},
\end{aligned}
$$

where $\operatorname{tr}_{+}[\cdots]=\frac{1}{2} \operatorname{tr}\left[\left(1+\gamma_{5}\right) \cdots\right]$. To simplify the expression we used,

$$
l_{1}^{2}=0, \quad l_{1} l_{3}=l_{1}\left(k_{3}+\not k_{4}\right), \quad l_{3} l_{1}=-\left(k_{1}+\not k_{2}\right) l_{1} .
$$

The $\gamma_{5}$ term in the trace drops out because $k_{4}$ is linearly dependent on the other external momenta and drops out when contracted into the totally anti-symmetric Levi-Civita tensor.

Combining the equations and putting back the cut propagators, we find that

$$
\left.A_{4 ; 1}^{\mathcal{N}=4}\left(1^{-}, 2^{-}, 3^{+}, 4^{+}\right)\right|_{s^{-c u t}}=-\left.s t A_{4}^{\text {tree }} I_{4}(s, t)\right|_{s^{-c u t}},
$$

has the correct $s$-channel cut, where $I_{4}(s, t)$ is the box integral,

$$
I_{4}(s, t)=-i \int \frac{d^{4-2 \epsilon} p}{(2 \pi)^{4-2 \epsilon}} \frac{1}{p^{2}\left(p-k_{1}\right)^{2}\left(p-k_{1}-k_{2}\right)^{2}\left(p+k_{4}\right)^{2}} .
$$

Now consider the $t$ channel shown in figure 1(b). In this case, all particles in the multiplet contribute to the cut. We label the states according to their helicity $h$. In this 
case the two tree-level amplitudes on either side of the cuts are given by

$$
\begin{aligned}
& A_{4}^{\text {tree }}\left(-l_{2}^{-h}, 2^{-}, 3^{+}, l_{4}^{h}\right)=i \frac{\left\langle-l_{2} 2\right\rangle^{2+2 h}\left\langle l_{4} 2\right\rangle^{2-2 h}}{\left\langle-l_{2} 2\right\rangle\langle 23\rangle\left\langle 3 l_{4}\right\rangle\left\langle l_{4}-l_{2}\right\rangle}, \\
& A_{4}^{\text {tree }}\left(-l_{4}^{-h}, 4^{+}, 1^{-}, l_{2}^{h}\right)=i \frac{\left\langle-l_{4} 1\right\rangle^{2+2 h}\left\langle l_{2} 1\right\rangle^{2-2 h}}{\left\langle-l_{4} 4\right\rangle\langle 41\rangle\left\langle 1 l_{2}\right\rangle\left\langle l_{2}-l_{4}\right\rangle} .
\end{aligned}
$$

In $\mathcal{N}=4$ super-Yang-Mills theory the helicity $h$ takes on values, $\{-1,-1 / 2,0,1 / 2,1\}$. These amplitudes can be obtained from the purely gluonic ones, using supersymmetry Ward Identities [63, 34] or by extracting the components from superspace amplitudes, discussed below.

The $t$-channel cut is given by multiplying the two amplitudes and summing over all intermediate states. This gives us

$$
C_{t}=-\rho \frac{1}{\left\langle-l_{2} 2\right\rangle\langle 23\rangle\left\langle 3 l_{4}\right\rangle\left\langle l_{4}-l_{2}\right\rangle} \frac{1}{\left\langle-l_{4} 4\right\rangle\langle 41\rangle\left\langle 1 l_{2}\right\rangle\left\langle l_{2}-l_{4}\right\rangle},
$$

where the factor $\rho$ contains the contributions from the gluon, four gluinos and six real scalars,

$$
\begin{aligned}
\rho= & \left(\left\langle l_{2} 2\right\rangle^{4}\left\langle l_{4} 1\right\rangle^{4}-4\left\langle l_{2} 2\right\rangle^{3}\left\langle l_{4} 2\right\rangle\left\langle l_{4} 1\right\rangle^{3}\left\langle l_{2} 1\right\rangle\right. \\
& +6\left\langle l_{2} 2\right\rangle^{2}\left\langle l_{4} 2\right\rangle^{2}\left\langle l_{4} 1\right\rangle^{2}\left\langle l_{2} 1\right\rangle^{2}-4\left\langle l_{2} 2\right\rangle\left\langle l_{4} 2\right\rangle^{3}\left\langle l_{2} 1\right\rangle^{3}\left\langle l_{4} 1\right\rangle \\
& \left.+\left\langle l_{4} 2\right\rangle^{4}\left\langle l_{2} 1\right\rangle^{4}\right) \\
= & \left(\left\langle l_{2} 2\right\rangle\left\langle l_{4} 1\right\rangle-\left\langle l_{4} 2\right\rangle\left\langle l_{2} 1\right\rangle\right)^{4} \\
= & \langle 12\rangle^{4}\left\langle l_{4} l_{2}\right\rangle^{4} .
\end{aligned}
$$

To obtain the final line we made use of a Schouten identity [64]. Thus, the $t$ channel cut collapses to a relabeling of the $s$ channel cut (16).

Following similar algebra as for the $s$ channel cut we obtain

$$
\left.A_{4}^{\mathcal{N}=4}\left(1^{-}, 2^{-}, 3^{+}, 4^{+}\right)\right|_{t \text {-cut }}=-\left.s t A_{4}^{\text {tree }} I_{4}(s, t)\right|_{t \text {-cut }},
$$

At this point, from eqs. (16) and (21) it is clear that the correct amplitude must be

$$
A_{4}^{\mathcal{N}=4}\left(1^{-}, 2^{-}, 3^{+}, 4^{+}\right)=-s t A_{4}^{\text {tree }} I_{4}(s, t),
$$

because it has the correct cuts in both the $s$ and $t$ channels. In this case the merging procedure for the cuts turns out to be trivial because the numerator does not contain loop momentum.

More complex cases both at one and higher loops are discussed in other chapters of this review [14, 13, 15]. Recent progress has also been made using cuts with only a single cut propagator [47, 37].

\section{Sewing $\mathcal{N}=4$ Super-Yang-Mills amplitudes}

For problems involving supersymmetric amplitudes, it is usually best to use an on-shell superspace which organizes the amplitudes according to physical helicity states. This 
provides a convenient means for dealing with all states of the theory simultaneously, and for carrying out intermediate sums of states crossing cuts. The superspace we discuss here consists of unconstrained Grassmann variables which form a fundamental representation of the $\mathrm{SU}(\mathcal{N})$ R-symmetry group. The unconstrained nature of the variables makes it simple to translate summations of on-shell states needed in unitarity cuts into Grassmann integrations, which take care of the bookkeeping.

\subsection{Tree-level superamplitudes}

We begin with a brief setup of the formalism. (See also the chapter of this review from Brandhuber, Spence and Travaglini [60].) The on-shell superspace is constructed by introducing a set of fermionic variables $\eta^{a}$, with $a=1 \cdots \mathcal{N}$ transforming in the fundamental representation of $\mathrm{SU}(\mathcal{N})$ R-symmetry. The bosonic spinor variables carry kinematic information, while the fermionic variables carry information on the helicity and R-symmetry representation of the external states. $\mathcal{N}=4$ super-Yang-Mills has a simple structure because all states can be incorporated into a self CPT superfield,

$$
\Phi(\eta)=g^{+}+\eta^{a} f_{a}^{+}+\frac{1}{2} \eta^{a} \eta^{b} \phi_{a b}+\frac{1}{3 !} \epsilon_{a b c d} \eta^{a} \eta^{b} \eta^{c} f^{d-}+\frac{1}{4 !} \epsilon_{a b c d} \eta^{a} \eta^{b} \eta^{c} \eta^{d} g^{-} .
$$

We note that $\mathcal{N}=8$ supergravity is similar, with a self CPT multiplet in four dimensions containing fields up to spin 2 .

These fermionic variables were first introduced by Ferber [66] to extend twistors, which are representations of four-dimensional conformal group, to supertwistors. Nair [61] applied these variables to represent MHV amplitudes of $\mathcal{N}=4$ super-YangMills theory. These amplitudes take the form

$$
\mathcal{A}_{n}^{\mathrm{MHV}}(1,2, \cdots, n)=\frac{i}{\prod_{j=1}^{n}\langle j(j+1)\rangle} \delta^{(8)}\left(\sum_{j=1}^{n} \lambda_{j}^{\alpha} \eta_{j}^{a}\right),
$$

where leg $n+1$ is to be identified with leg 1 , and

$$
\delta^{(8)}\left(\sum_{j=1}^{n} \lambda_{j}^{\alpha} \eta_{j}^{a}\right)=\prod_{a=1}^{4} \sum_{i<j}^{n}\langle i j\rangle \eta_{i}^{a} \eta_{j}^{a} .
$$

The component amplitudes are the coefficients in the $\eta$ expansion of $\mathcal{A}_{n}$, with the external states identified according to their organization within the superfield as in eq. (23).

An MHV superamplitude of $\mathcal{N}=4$ super-Yang-Mills can also be written as

$$
\mathcal{A}_{n}^{\mathrm{MHV}}=\frac{A_{n}^{\text {tree }}\left(1^{-}, 2^{-}, 3^{+}, \cdots, n^{+}\right)}{\langle 12\rangle^{4}} \delta^{(8)}\left(\sum_{j=1}^{n} \lambda_{j}^{\alpha} \eta_{j}^{a}\right),
$$

where $A_{n}^{\text {tree }}\left(1^{-}, 2^{-}, 3^{+}, \cdots, n^{+}\right)$is the tree-level MHV pure-gluon amplitude. We note that the MHV superamplitudes of $\mathcal{N}=8$ supergravity has a similar form

$$
\mathcal{M}_{n}^{\mathrm{MHV}}=\frac{M_{n}\left(1^{-}, 2^{-}, 3^{+}, \cdots, n^{+}\right)}{\langle 12\rangle^{8}} \delta^{(16)}\left(\sum_{j=1}^{n} \lambda_{j}^{\alpha} \eta_{j}^{a}\right)
$$


where $M_{n}\left(1^{-}, 2^{-}, 3^{+}, \cdots, n^{+}\right)$is a tree-level MHV pure-graviton amplitude.

It is natural to expect that superamplitudes for theories with fewer supersymmetries are simply given by subamplitudes of the maximal theory. Indeed as shown in ref. [58], by grouping R-symmetry indices, one obtains amplitudes for the gauge multiplet of lower supersymmetric theories. The most trivial example is that the amplitudes of pure non-supersymmetric Yang-Mills theory are just the pure gluon amplitudes of $\mathcal{N}=4$ super-Yang-Mills theory, since all other states decouple from these amplitudes. In fact, by making appropriate projections, one can even obtain QCD amplitudes with quarks from $\mathcal{N}=4$ tree amplitudes [67], leading to compact forms of QCD amplitudes.

As a simple example, the MHV tree amplitudes for the minimal gauge multiplets of $\mathcal{N}<4$ super-Yang-Mills theory are given by [58]

$$
\mathcal{A}_{n}^{\mathrm{MHV}}(1,2, \ldots, n)=\frac{\prod_{a=1}^{\mathcal{N}} \delta^{(2)}\left(Q^{a}\right)}{\prod_{j=1}^{n}\langle j(j+1)\rangle}\left(\sum_{i<j}^{n}\langle i j\rangle^{4-\mathcal{N}} \prod_{a=\mathcal{N}+1}^{4} \eta_{i}^{a} \eta_{j}^{a}\right),
$$

with $\mathcal{N}$ counting the number of supersymmetries, $Q^{a}=\sum_{i=1}^{n} \lambda_{i} \eta_{i}^{a}$, and $n \geq 3$. As we discuss below, these can be used as input building blocks to construct the integrands of loop superamplitudes. For a recent discussion on $\mathcal{N}<4$ superamplitudes, see [68].

The parity conjugate $\overline{\mathrm{MHV}}$ superamplitude is simply given by exchanging $\lambda_{i}^{\alpha} \leftrightarrow \tilde{\lambda}_{i}^{\dot{\alpha}}$ and $\eta_{i}^{a} \leftrightarrow \tilde{\eta}_{i a}$. To obtain the component amplitude from the $\overline{\mathrm{MHV}}$ superamplitude, one can perform a Grassmann Fourier transformation to convert it back to the $\eta$ representation [57]. Starting from the three-point MHV and $\overline{\mathrm{MHV}}$ amplitude, one can obtain the higher-point amplitudes in superspace via the MHV vertex expansion constructed by Cachazo, Svrček and Witten (CSW) 69] or the on-shell recursion relations of Britto, Cachazo, Feng, and Witten (BCFW) [7, 70]. The supersymmetric extension of the former approach is given in refs. $71,56,57,58$, while that of the latter is given in refs. [49, 23, 25, 72].

The expression for the $\mathrm{N}^{m} \mathrm{MHV}$ superamplitude constructed via the CSW construction is given by

$$
\mathcal{A}_{n}^{\mathrm{N}^{m} \mathrm{MHV}}=i^{m} \sum_{\mathrm{CSW} \text { graphs }} \int\left[\prod_{j=1}^{m} \frac{d^{4} \eta_{j}}{P_{j}^{2}}\right] \mathcal{A}_{(1)}^{\mathrm{MHV}} \mathcal{A}_{(2)}^{\mathrm{MHV}} \cdots \mathcal{A}_{(m)}^{\mathrm{MHV}} \mathcal{A}_{(m+1)}^{\mathrm{MHV}},
$$

where the integral is over the $4 m$ internal Grassmann parameters $\left(d^{4} \eta_{j} \equiv \prod_{a=1}^{4} d \eta_{j}^{a}\right)$ associated with the internal legs, and each $P_{j}$ is the momentum of the $j$ 'th internal leg of the graph. To each vertex one associates an on-shell MHV superamplitude, and the holomorphic spinor $\lambda_{P_{j}}$, also denoted as $\left|P_{j}^{b}\right\rangle$, associated to an internal leg is constructed from the corresponding off-shell momentum via $P_{j}^{b}=P_{j}-r P_{j}^{2} / 2 P_{j} \cdot r$, where $r$ is a null reference momentum [73].

Alternatively, the $n$-point $\mathrm{N}^{m} \mathrm{MHV}$ tree level superamplitudes can be obtained via the super-BCFW recursion relations. An advantage of this approach is that the final form of the amplitude can be given so that dual conformal symmetry is manifest [25]

‡ The amplitudes are actually dual superconformal covariant, not invariant. This covariance can be translated into an invariance, by considering the Yangian generators which are obtained by combining 
This symmetry [20, 23] is a conformal symmetry defined in the dual space $x$, which is related to the external momenta via

$$
x_{i}-x_{i+1}=p_{i},
$$

where $x_{n+1} \equiv x_{1}$. (Further details on dual (super)conformal symmetry may be found in the chapters of the present review by Drummond and by Henn [28, 29].) Using super$\mathrm{BCFW}$ recursion relations, Drummond and Henn were able to derive all $\mathrm{N}^{m} \mathrm{MHV}$ tree amplitudes in terms of dual conformal invariants in ref. [72]. For example, the $n$-point NMHV amplitude is given as

$$
\mathcal{A}_{n}^{\mathrm{NMHV}}(1,2, \cdots, n)=\mathcal{A}_{n}^{\mathrm{MHV}} \sum_{1<s, t<n} R_{n ; s t},
$$

where the function $R_{n ; s t}$ is a dual conformal invariant function. The specific form, and its $\mathrm{N}^{m} \mathrm{MHV}$ generalization may be found in ref. [72, 29].

The tree amplitudes obtained from the $\mathrm{BCFW}$ or $\mathrm{CSW}$ procedure in general contain spurious poles which cancel between different contributions. The spurious poles complicate the merging procedure of different cuts. However, if one works in the reverse direction starting from an ansatz, one can use numerical analysis to solve for coefficients of the ansatz for the amplitude. In this way, complications from the spurious poles can be easily avoided. (At one loop see also the discussion in Britto's chapter of the review [13].)

\subsection{Loop superamplitudes}

We now turn to the construction of four-dimensional unitarity cuts in superspace. The sum over states crossing the unitarity cuts can be expressed simply as an integration over the $\eta^{a}$ parameters of the cut legs, since this gives the states properly on either side of the cut. The generalized $\mathcal{N}=4$ supercut is then given by

$$
\mathcal{C}=\int\left[\prod_{i=1}^{k} d^{4} \eta_{i}\right] \mathcal{A}_{(1)}^{\text {tree }} \mathcal{A}_{(2)}^{\text {tree }} \mathcal{A}_{(3)}^{\text {tree }} \cdots \mathcal{A}_{(m)}^{\text {tree }},
$$

where $\mathcal{A}_{(j)}^{\text {tree }}$ are the tree superamplitudes connected by $k$ on-shell cut legs. These cuts then constrain the amplitude, which are now functions in the on-shell superspace.

For four and higher points the tree amplitude $\mathcal{A}_{(j)}^{\text {tree }}$ is always proportional to a supermomentum delta functions. Using the identity $\delta(A) \delta(B)=\delta(A+B) \delta(B)$, this implies that all such cuts are proportional to an overall supermomentum delta function [21]. As discussed in section 2, a spanning set of cuts can be constructed using four and higher-point tree amplitudes. This then implies that any loop amplitude with four or more legs must be proportional to an overall supermomentum conservation delta function. One interesting consequence is that this overall delta function implies that at least four would be powers of loop momenta in the numerators of each diagram are ordinary and dual superconformal generators [74, 75].

$\S$ The exception is the three-point $\overline{\mathrm{MHV}}$ superamplitude where the supermomentum conservation is not manifest [21]. 
external momenta. Simple power counting then implies the well known [76] ultraviolet finiteness of all $\mathcal{N}=4$ super-Yang-Mills amplitudes [58].

There are a number of ways of evaluating supersums in unitarity cuts [21, 49, 57, 58]. Here we describe two such approaches for organizing the integration over the Grassmann parameters. In the first way, the fermionic delta functions can be used to localize the integration, so that the evaluation of the supersum amounts to solving a subset of linear equations [21, 58]. In a second complementary approach one uses "index diagrams" to track the contributions [58]. This latter approach leads to a simple algorithm for reading off the contribution of the entire supermultiplet from the purely gluonic ones or for reducing supersymmetry and was used in the construction of the complete four-loop four-point amplitude of $\mathcal{N}=4$ super-Yang-Mills theory [11].

\subsection{The supersum as a system of linear equations}

We now consider in more detail the evaluation of supersums. First consider the approach based on solving a system of equations. This task is straightforward if the product of tree amplitudes is written in a form that depends on the $\eta^{a}$ s only through fermionic delta functions. The MHV vertex expansion offers a simple way to accomplish this [69]. The arguments of the delta functions then automatically form a set of linear equations that constrain the $\eta^{a}$ s.

Simple counting shows that after the overall supermomentum conservation constraint is extracted, the number of equations appearing in cuts of MHV loop amplitudes equals the number of integration variables. For such cuts the integrals are completely localized by delta functions, and one simply computes the Jacobian of the linear equations.

For $\mathrm{N}^{m} \mathrm{MHV}$ amplitudes, the number of constraints will be larger than the number of integration variables. For this case, one proceeds by solving some judiciously chosen subset of the supermomentum constraints and substituting the result into the remaining fermionic delta functions. However, some choices of solving subsets of the constraints may lead to results that obscure symmetries of the amplitude. One simple helpful strategy is to choose the constraints with as few external momenta as possible.

4.3.1. Example: The one-loop four-point amplitude. To illustrate these ideas we turn to a few simple examples. As a first example, we revisit the computation of the oneloop four-point amplitude of $\mathcal{N}=4$ super-Yang-Mills theory. Consider the $s$-channel supercut of the one-loop four-point superamplitude shown in figure 1(a). The supersum is obtained from the Grassmann integrals,

$$
\mathcal{C}_{s}=\int d^{4} \eta_{l_{1}} \int d^{4} \eta_{l_{3}} \mathcal{A}_{4}^{\mathrm{MHV}}\left(-l_{1}, 1,2, l_{3}\right) \mathcal{A}_{4}^{\mathrm{MHV}}\left(-l_{3}, 3,4, l_{1}\right),
$$

where the MHV superamplitudes are given by relabeling eq. (24) for $n=4$. The $\eta$ integration acts on the two supermomentum delta functions contained in the tree 
superamplitudes,

$$
\delta^{(8)}\left(\lambda_{l_{3}}^{\alpha} \eta_{l_{3}}^{a}-\lambda_{l_{1}}^{\alpha} \eta_{l_{1}}^{a}+\lambda_{1}^{\alpha} \eta_{1}^{a}+\lambda_{2}^{\alpha} \eta_{2}^{a}\right) \delta^{(8)}\left(\lambda_{l_{1}}^{\alpha} \eta_{l_{1}}^{a}-\lambda_{l_{3}}^{\alpha} \eta_{l_{3}}^{a}+\lambda_{3}^{\alpha} \eta_{3}^{a}+\lambda_{4}^{\alpha} \eta_{4}^{a}\right)
$$

Adding the argument of the first delta function to the second one, one obtains an overall supermomentum conservation delta function that can be extracted outside of the Grassmann integral,

$$
\mathcal{C}_{s}=-\delta^{(8)}\left(\sum_{i=1}^{4} \lambda_{i}^{\alpha} \eta_{i}^{a}\right) \frac{\int d^{4} \eta_{l_{1}} d^{4} \eta_{l_{3}} \delta^{(8)}\left(\lambda_{l_{3}}^{\alpha} \eta_{l_{3}}^{a}-\lambda_{l_{1}}^{\alpha} \eta_{l_{1}}^{a}+\lambda_{1}^{\alpha} \eta_{1}^{a}+\lambda_{2}^{\alpha} \eta_{2}^{a}\right)}{\left\langle l_{1} 1\right\rangle\langle 12\rangle\left\langle 2 l_{3}\right\rangle\left\langle l_{3} l_{1}\right\rangle\left\langle l_{3} 3\right\rangle\langle 34\rangle\left\langle 4 l_{1}\right\rangle\left\langle l_{1} l_{3}\right\rangle}
$$

Choosing an explicit value for the R-symmetry index, for example $a=1$, the fermionic integration becomes,

$$
\int d \eta_{l_{1}}^{a} d \eta_{l_{3}}^{a} \delta^{(2)}\left(\lambda_{l_{3}}^{\alpha} \eta_{l_{3}}^{a}-\lambda_{l_{1}}^{\alpha} \eta_{l_{1}}^{a}+\lambda_{1}^{\alpha} \eta_{1}^{a}+\lambda_{2}^{\alpha} \eta_{2}^{a}\right)=-\left\langle l_{3} l_{1}\right\rangle
$$

which may be read off using the form of the delta function displayed in eq. (25). Similarly, the cases $a=2,3,4$ give the identical factor. Thus, the entire fermionic integration simply gives a factor of $\left\langle l_{3} l_{1}\right\rangle^{4}$.

Alternatively, the fermionic delta function inside the Grassmann integral in eq. (35) can be viewed as enforcing the constraints,

$$
\lambda_{l_{1}}^{\alpha} \eta_{l_{1}}^{a}-\lambda_{l_{3}}^{\alpha} \eta_{l_{3}}^{a}=\lambda_{1}^{\alpha} \eta_{1}^{a}+\lambda_{2}^{\alpha} \eta_{2}^{a} .
$$

There are a total of eight conditions which matches the eight integration variables $\eta_{l_{1}}^{a}, \eta_{l_{3}}^{a}$. Thus, the delta functions completely fix the integration and one obtains the Jacobian of the matrix of the coefficients of the linear equations,

$$
J=\operatorname{det}^{4}\left|\begin{array}{cc}
\lambda_{l_{1}}^{1} & -\lambda_{l_{3}}^{1} \\
\lambda_{l_{1}}^{2} & -\lambda_{l_{3}}^{2}
\end{array}\right|=\left\langle l_{1} l_{3}\right\rangle^{4} .
$$

Either way, the $s$-channel cut of the superamplitude is

$$
\mathcal{C}_{s}=-\frac{\delta^{(8)}\left(\sum_{i=1}^{n} \lambda_{i}^{\alpha} \eta_{i}^{a}\right)\left\langle l_{1} l_{3}\right\rangle^{4}}{\left\langle l_{1} 1\right\rangle\langle 12\rangle\left\langle 2 l_{3}\right\rangle\left\langle l_{3} l_{1}\right\rangle\left\langle l_{3} 3\right\rangle\langle 34\rangle\left\langle 4 l_{1}\right\rangle\left\langle l_{1} l_{3}\right\rangle} .
$$

Other than the replacement of a factor of $\langle 12\rangle^{4}$ by the overall supermomentum delta function, this is the same expression as arrived at by using components as in eq. (10). Thus, following the same steps as for components one obtains

$$
\mathcal{C}_{s}=\mathcal{A}_{4}^{\text {tree }}(1,2,3,4) \frac{-i s t}{\left(p-k_{1}\right)^{2}\left(p+k_{4}\right)^{2}} .
$$

Putting back the cut propagators and comparing with the $t$-channel cut, one arrives at the four-point one-loop superamplitude,

$$
\mathcal{A}_{4}^{1-\text { loop }}=-s t \mathcal{A}_{4}^{\text {tree }} I_{4}(s, t),
$$

where the box integral $I_{4}(s, t)$ is defined in eq. (17). This result is in agreement with the component analysis of section 3 . 


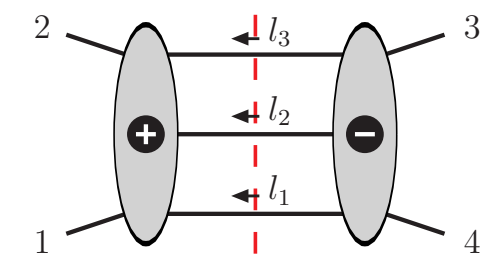

Figure 5. A three-particle supercut for the four-point amplitude. This cut contribution contains one MHV and one $\overline{\mathrm{MHV}}$ superamplitude. The "+" indicates the MHV amplitude, while the "-" indicates the $\overline{\mathrm{MHV}}$ one.

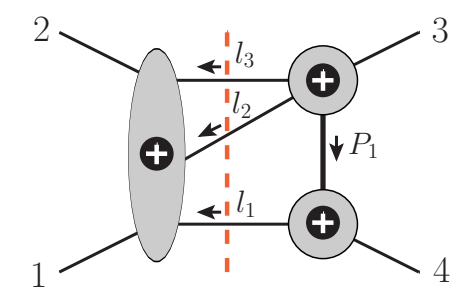

Figure 6. The same cut as in figure 5 , except that $\overline{\mathrm{MHV}}$ tree amplitude on the right is expanded in terms of MHV amplitudes. We display only one of the terms here. The thick line labeled by $P$ marks the internal propagator used in the MHV expansion.

4.3.2. Example: The two-loop four-point amplitude using the MHV expansion. As a more sophisticated example, consider the three-particle cut of the two-loop four-gluon amplitude displayed in figure 5. This cut contains both MHV and $\overline{M H V}$ amplitudes. This cut was evaluated in components in refs. [48, 50] and in superspace in ref. [57, 58]. The supercut of figure 5 may be conveniently evaluated using a Grassmann Fourier transform. Instead, for illustrative purposes here we expand the $\overline{\mathrm{MHV}}$ tree amplitude using the MHV vertex expansion [69]. (See the chapter in this review 60] giving further details on the MHV expansion.) This procedure provides a general means for evaluating any cut not involving $\overline{\mathrm{MHV}}$ three-point tree amplitudes. $\llbracket$ The MHV vertex expansion reduces any generalized cut to cuts involving only MHV amplitudes. We thus expand the $\overline{\mathrm{MHV}}$ tree amplitude on the right side of the cut in figure 5. One of the obtained terms in the MHV expansion is shown figure 6. Here we will describe how to obtain the result for this specific supercut contribution; the remaining ones are similar.

For the cut in figure 6, for a fixed R-symmetry index $a$ we have three supermomentum constraints from each of the three MHV amplitudes,

$$
\begin{aligned}
& \delta^{(2)}\left(\lambda_{1}^{\alpha} \eta_{1}^{a}+\lambda_{2}^{\alpha} \eta_{2}^{a}-\lambda_{l_{1}}^{\alpha} \eta_{l_{1}}^{a}-\lambda_{l_{2}}^{\alpha} \eta_{l_{2}}^{a}-\lambda_{l_{3}}^{\alpha} \eta_{l_{3}}^{a}\right) \\
& \times \delta^{(2)}\left(\lambda_{3}^{\alpha} \eta_{3}^{a}+\lambda_{P_{1}}^{\alpha} \eta_{P_{1}}^{a}+\lambda_{l_{2}}^{\alpha} \eta_{l_{2}}^{a}+\lambda_{l_{3}}^{\alpha} \eta_{l_{3}}^{a}\right) \\
& \times \delta^{(2)}\left(\lambda_{4}^{\alpha} \eta_{4}^{a}+\lambda_{l_{1}}^{\alpha} \eta_{l_{1}}^{a}-\lambda_{P_{1}}^{\alpha} \eta_{P_{1}}^{a}\right) .
\end{aligned}
$$

In this expression $l_{1}, l_{2}$ and $l_{3}$ refer to the momenta of the three cut lines. The spinors labeled by $P_{1}$ satisfy $\lambda_{P_{1}} \tilde{\lambda}_{P_{1}}=P_{1}^{\mathrm{b}}=P_{1}-r P_{1}^{2} / 2 P_{1} \cdot r$, with $r$ a null reference momentum

\|If three-point $\overline{\mathrm{MHV}}$ tree amplitudes are present in a given cut, we can either absorb them into cuts with higher-point trees, or apply Fourier transform techniques [57, 58, 
and $P_{1}=l_{1}+k_{4}$, as arises in the MHV expansion [69, 73]. We first extract the overall supermomentum conservation delta function and leave behind the second and third delta function. These enforce the constraints,

$$
\begin{aligned}
-\lambda_{P_{1}}^{\alpha} \eta_{P_{1}}^{a}-\lambda_{l_{2}}^{\alpha} \eta_{l_{2}}^{a}-\lambda_{l_{3}}^{\alpha} \eta_{l_{3}}^{a} & =\lambda_{3}^{\alpha} \eta_{3}^{a}, \\
+\lambda_{P_{1}}^{\alpha} \eta_{P_{1}}^{a}-\lambda_{l_{1}}^{\alpha} \eta_{l_{1}}^{a} & =\lambda_{4}^{\alpha} \eta_{4}^{a} .
\end{aligned}
$$

Since there are 16 equations for $4 \times 4=16$ variables, the integrals are completely localized and one simply needs the Jacobian of the matrix of coefficients,

$$
\left(\begin{array}{cccc}
-\lambda_{P_{1}}^{\alpha} & 0 & -\lambda_{l_{2}}^{\alpha} & -\lambda_{l_{3}}^{\alpha} \\
+\lambda_{P_{1}}^{\alpha} & -\lambda_{l_{1}}^{\alpha} & 0 & 0
\end{array}\right)
$$

where each spinor $\lambda_{j}^{\alpha}$ should be thought of as a submatrix with two rows and one column. The Jacobian of this matrix is just $\left\langle l_{1} P_{1}^{b}\right\rangle\left\langle l_{2} l_{3}\right\rangle$. For each of the four values that the R-symmetry index takes on we get the same result. Thus the Grassmann integration over the delta functions in eq. (42) evaluates to,

$$
\left(\left\langle l_{1} P_{1}^{b}\right\rangle\left\langle l_{2} l_{3}\right\rangle\right)^{4} \delta^{(8)}\left(\lambda_{1}^{\alpha} \eta_{1}^{a}+\lambda_{2}^{\alpha} \eta_{2}^{a}+\lambda_{3}^{\alpha} \eta_{3}^{a}+\lambda_{4}^{\alpha} \eta_{4}^{a}\right) .
$$

To obtain the complete result, one needs to repeat the same steps for other diagrams corresponding to the other terms in the MHV expansion of the five-point $\overline{\mathrm{MHV}}$ amplitude. Although the result is overly complicated because of the expansion of a simple $\overline{\mathrm{MHV}}$ tree amplitude in MHV diagrams, it does illustrate a general technique for evaluating supersums in unitarity cuts.

4.3.3. Example: The two-loop four-point amplitude using the $N^{m} M H V$ dual conformal representation. As another means for evaluating generalized cuts in $\mathcal{N}=4$ super-YangMills theory, one can start from the closed form $\mathrm{N}^{m} \mathrm{MHV}$ superamplitudes derived in ref. [72]. (These tree solutions also may be in found in the chapter of this review from Drummond [28].) One proceeds by using the fermionic delta functions to localize part of the integral, and implementing the solution of the constraints into the remaining superfunction. The final integrals then pick out specific pieces of the superfunction.

More concretely, consider again the tree amplitude on the right-hand-side of the three-particle cut in figure 5 as an NMHV superamplitude. The supermomentum delta functions can be used to localize a total of two loop $\eta^{a}$ S for each SU(4) R-index. Take them to be $l_{1}$ and $l_{2}$. The supersum can then be rewritten as

$$
\int\left[\prod_{i=1}^{3} d \eta_{l_{i}}^{a}\right] \delta^{(2)}\left(Q_{L}^{a}\right) \delta^{(2)}\left(Q_{R}^{a}\right)\left(R_{3 ; l_{1} l_{2}}+R_{3 ; l_{3} l_{2}}+R_{3 ; l_{3} l_{1}}\right),
$$

where

$$
\begin{aligned}
& Q_{L}^{\alpha a}=\lambda_{1}^{\alpha} \eta_{1}^{a}+\lambda_{2}^{\alpha} \eta_{2}^{a}-\lambda_{l_{1}}^{\alpha} \eta_{l_{1}}^{a}-\lambda_{l_{2}}^{\alpha} \eta_{l_{2}}^{a}-\lambda_{l_{3}}^{\alpha} \eta_{l_{3}}^{a}, \\
& Q_{R}^{\alpha a}=\lambda_{3}^{\alpha} \eta_{3}^{a}+\lambda_{4}^{\alpha} \eta_{4}^{a}+\lambda_{l_{1}}^{\alpha} \eta_{l_{1}}^{a}+\lambda_{l_{2}}^{\alpha} \eta_{l_{2}}^{a}+\lambda_{l_{3}}^{\alpha} \eta_{l_{3}}^{a},
\end{aligned}
$$

and the $R$ s are a set of dual conformal invariant functions mentioned in section 4.1 , Pulling out the overall supermomentum delta function and leaving behind $\delta^{(2)}\left(Q_{R}^{a}\right)$, we 
can rewrite eq. (46) as

$$
\begin{aligned}
\left\langle l_{1} l_{2}\right\rangle \delta^{(2)}\left(\sum_{i=1}^{4} q_{i}^{a}\right) & \int\left[\prod_{i=1}^{3} d \eta_{l_{i}}^{a}\right] \delta\left(\eta_{l_{1}}^{a}+\frac{\left\langle l_{2}\right|\left(q_{l_{3}}^{a}+q_{3}^{a}+q_{4}^{a}\right)}{\left\langle l_{2} l_{1}\right\rangle}\right) \\
& \times \delta\left(\eta_{l_{2}}^{a}+\frac{\left\langle l_{1}\right|\left(q_{l_{3}}^{a}+q_{3}^{a}+q_{4}^{a}\right)}{\left\langle l_{1} l_{2}\right\rangle}\right)\left(R_{3 ; l_{1} l_{2}}+R_{3 ; l_{3} l_{2}}+R_{3 ; l_{3} l_{1}}\right) .
\end{aligned}
$$

Thus, the delta functions simply localizes the $\eta_{l_{1}}^{a}, \eta_{l_{2}}^{a}$ integral and we obtain

$$
\left.\delta^{(2)}\left(\sum_{i=1}^{4} q_{i}^{a}\right) \int d \eta_{l_{3}}^{a}\left\langle l_{1} l_{2}\right\rangle\left(R_{3 ; l_{1} l_{2}}+R_{3 ; l_{3} l_{2}}+R_{3 ; l_{3} l_{1}}\right)\right|_{\text {sol }},
$$

where $\left.\left(\cdots+R_{3 ; l_{3} l_{1}}\right)\right|_{\text {sol }}$ signifies that all $\eta_{l_{1}}^{a}, \eta_{l_{2}}^{a}$ in the functions $R_{n ; s t}$ have been set to the solution of the fermionic constraints,

$$
\eta_{l_{1}}^{a}=-\frac{\left\langle l_{2}\right|\left(q_{l_{3}}^{a}+q_{3}^{a}+q_{4}^{a}\right)}{\left\langle l_{2} l_{1}\right\rangle}, \quad \eta_{l_{2}}^{a}=-\frac{\left\langle l_{1}\right|\left(q_{l_{3}}^{a}+q_{3}^{a}+q_{4}^{a}\right)}{\left\langle l_{1} l_{2}\right\rangle} .
$$

The final $\eta_{l_{3}}^{a}$ integration then simply picks out terms proportional to $q_{l_{3}}^{a}$ in the final expression.

In all of the above examples, after pulling out the overall supermomentum delta function, all fermionic variables are integrated away in the final expression. This property is unique to MHV loop amplitudes. For $\mathrm{N}^{m} \mathrm{MHV}$ amplitudes, there will be $4 m$ fermionic variables left after performing all Grassmann integrations. A convenient way of organizing these variables is given in ref. [54]. For planar amplitudes in the $\mathcal{N}=4$ theory, the presence of dual conformal symmetry implies that these variables can be organized into the invariant function $R_{n ; s t}$ and its generalizations.

\subsection{Supersums as $S U(4)$ index diagrams}

As manifest in the above examples, evaluations of the supersums appearing in cuts of $\mathcal{N}=4$ super-Yang-Mills theory boil down to evaluating the $\eta_{a}$ integration for a fixed $\mathrm{SU}$ (4) R-index, and taking the result to the fourth power. In this section, we explain this structure by keeping track of the different contributing states using a diagrammatic language to track the R-symmetry indices [58]. This leads to a surprisingly simple algorithm for essentially reading off contributions to the supercuts starting from nonsupersymmetric purely gluonic cuts. This algorithm provided a simple means to evaluate the supersums in the large number of generalized cuts providing cross checks in the calculation of the four-loop four-point amplitudes of $\mathcal{N}=4$ super-Yang-Mills theory [11] and $\mathcal{N}=8$ supergravity [31. Because individual states are tracked, the diagrammatic language also easily allows us to reduce the supersymmetry.

$$
\stackrel{a}{\stackrel{a}{:}} \quad \stackrel{a}{i} \eta_{i}^{a}\langle i j\rangle \eta_{i}^{a}
$$

Figure 7. For an MHV amplitude the shaded (blue) "index line" connecting leg $i$ to leg $j$ represents $\left\langle q_{i}^{a} q_{j}^{a}\right\rangle$. 
4.4.1. Structure of index diagrams. To see how the index diagrams arise, we first look at tree amplitudes. Up to the fact that all fields in the $\mathcal{N}=4$ multiplet are in the adjoint color representation, one sees that the $\mathrm{SU}(4) \mathrm{R}$ symmetry is analogous to the $\mathrm{SU}\left(N_{f}\right)$ flavor symmetry of QCD. Much like QCD, where flavor lines must be conserved within the amplitude, one can construct R-symmetry conserving lines for $\mathcal{N}=4$ super-Yang-Mills theory. This is most transparent for MHV tree amplitudes, where the supermomentum delta function can be considered as four copies of

$$
\left\langle q_{i}^{a} q_{j}^{a}\right\rangle \equiv \eta_{i}^{a}\langle i j\rangle \eta_{j}^{a} .
$$

The pair of external legs $(i, j)$ associated with each $\mathrm{R}$ index can be different. Pictorially, we can represent the supermomentum product by a shaded (blue) line connecting legs $i$ and $j$, as displayed figure 7 . We will call this object an "index line". Since different component amplitudes corresponds to different R-index structures for the external legs, in terms of index diagrams, this translate into different "paths" for the index lines. We illustrate the different component amplitudes of the four-point MHV amplitudes in terms of index diagrams in figure 8, Note that the pure gluon amplitudes corresponds to four index lines following the same path as in figure 8 (a). Amplitudes with fermions or scalars correspond to cases where index lines follow different paths as illustrated in figure 8 , We can also define index lines for $\overline{\mathrm{MHV}}$ amplitudes by switching the role of positive and negative helicity helicity spinors.

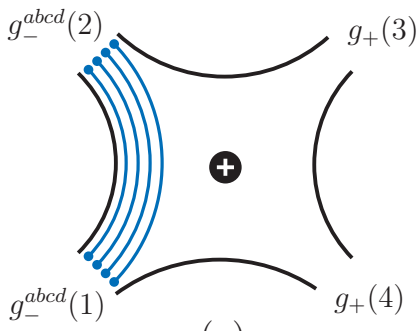

(a)

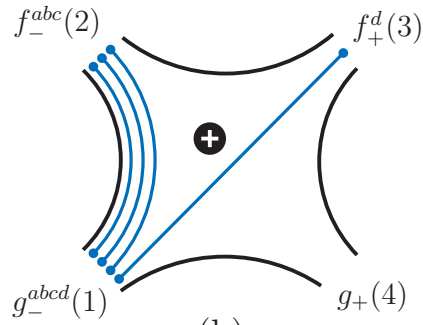

(b)

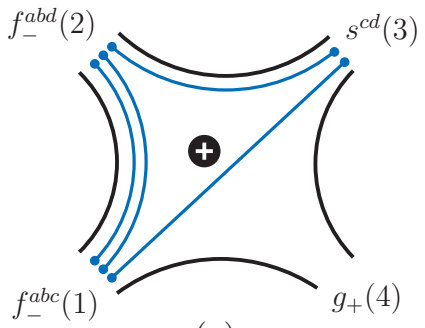

(c)

Figure 8. Examples of R-symmetry index diagrams for specifying different component amplitudes. Case (a) is a pure gluon amplitude, case (b) a twofermion two-gluon amplitude, and case (c) a two-fermion one-scalar one-gluon amplitude. The black outer lines connect the external legs and the shaded (blue) lines are the R-symmetry index lines. A white "+" and "-" on a black background indicates whether the amplitude is MHV or $\overline{\mathrm{MHV}}$.

The index line language generalizes straightforwardly to any tree amplitude via the MHV expansion [69]. In this expansion we obtain sums of products of on-shell tree amplitudes, but with shifted momenta. To obtain an index-diagram representation we simply dress each MHV component amplitude by the index diagrams. This diagrammatic representation also extends straightforwardly to loops. To do so we expand any component tree amplitude appearing in the cuts that is not MHV or $\overline{\mathrm{MHV}}$ into such amplitudes using the MHV expansion. If both $\mathrm{MHV}$ and $\overline{\mathrm{MHV}}$ tree amplitudes 


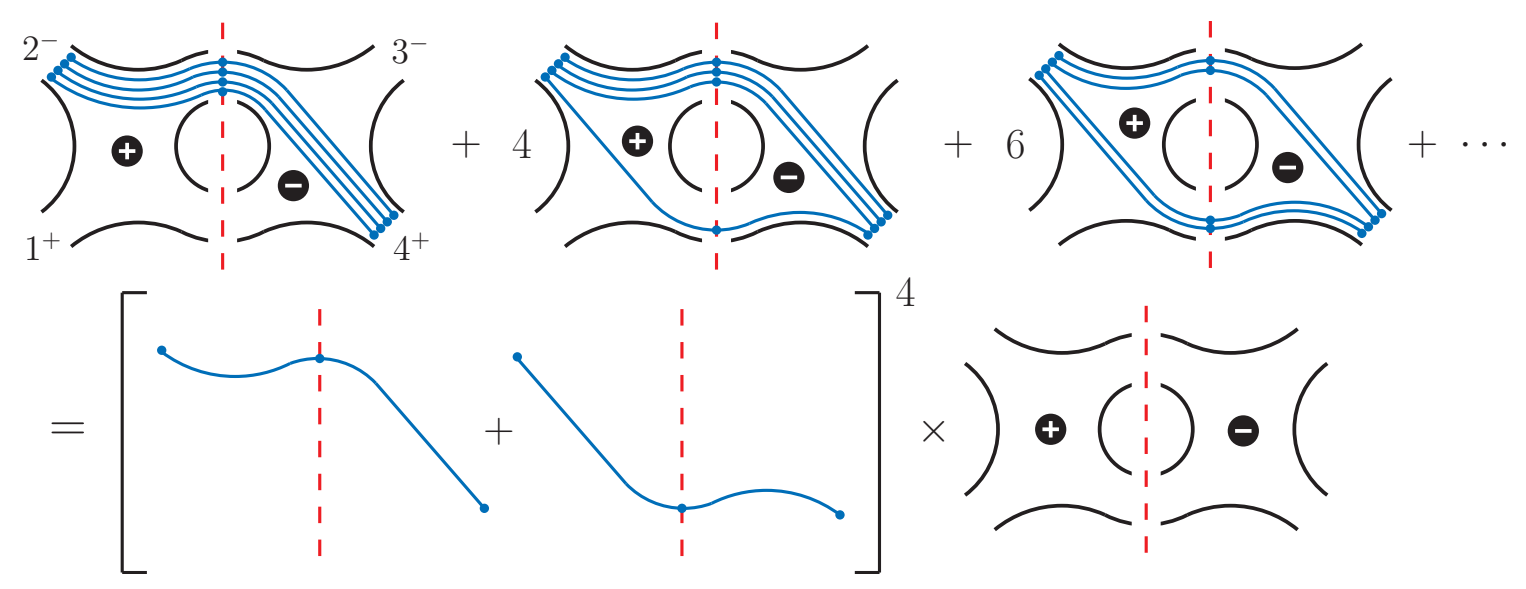

Figure 9. A unitarity cut of the four-gluon amplitude $A_{4}^{1-\text { loop }}\left(1^{+}, 2^{-}, 3^{-}, 4^{+}\right)$, involving one $\mathrm{MHV}$ and one $\overline{\mathrm{MHV}}$ superamplitude. The top-left diagram represents a gluon loop, the top-central diagram represents the four contributions in a fermion loop, and the top-right diagram represents the six scalar state contributions. The ellipsis denote that four more fermion-loop and one more gluon-loop contributions are suppressed.

are present in the cuts, the index lines can either cross, terminate or begin at the cuts. The details are given in ref. [58].

To illustrate the basic idea, we use the simple case of a cut of the one-loop fourpoint $\mathcal{N}=4$ amplitude, shown in figure 9. The left side of the cut is chosen to be MHV and right side is $\overline{\mathrm{MHV}}$. In this case, the $\mathrm{SU}(4)$ R-symmetry index lines are continuous through the cut. The different diagrams in the top line of figure 9 correspond to the different states crossing the cuts, the two hidden in the ellipsis are horizontal flips of the first two shown. The combinatoric factors in front of each diagram are the distinct ways of obtaining the same diagram, tracking the four distinct SU(4) labels.

As shown in figure 9, the sum over the diagrams can be interpreted as a product over the four R-symmetry indices, depicted as a fourth power. The pure gluon configuration then corresponds to all four index lines taking the same "path". Mixed paths then correspond to configurations with scalars and fermions. This structure, in fact, holds for any cut composed of MHV and $\overline{\mathrm{MHV}}$ amplitudes. Since the MHV expansion allows us to express any cut composed of tree amplitudes in terms of MHV and $\overline{\mathrm{MHV}}$ amplitude, for $\mathcal{N}=4$ super-Yang-Mills amplitudes all cuts are then given by a sum of terms, each with a numerator raised to the fourth power.

4.4.2. $\mathcal{N}=4$ loop amplitudes from pure gluon amplitudes. The one-loop discussion extends straightforwardly to multiloops [58]. By analyzing these index diagrams a simple pattern emerges: in the $\mathcal{N}=4$ theory for each configuration of MHV and $\overline{\mathrm{MHV}}$ amplitudes in a given cut, the supersums' contributions for external gluons are always proportional to the fourth power of a sum of terms,

$$
(A+B+C+\cdots)^{4},
$$


where the summands $A, B, C, \ldots$ represent different spinorial numerator factors encoded by the R-symmetry index diagrams. On the other hand, the pure gluon contribution takes the form

$$
\left(A^{4}+B^{4}+C^{4}+\cdots\right) \text {. }
$$

Comparing the two, we see that the supersum result (52) is identical to the pure gluon result (53), except the fourth power appears on the sum of terms instead of individual terms. Thus, we can read off the contributions of the entire $\mathcal{N}=4$ multiplet directly from the pure gluon contributions, up to relative signs. The relative signs between the terms in eq. (52) can be tracked by including Grassmann parameters. The details of this procedure are presented in ref. [58].

4.4.3. Amplitudes with fewer supersymmetries from $\mathcal{N}=4$ ones. As a further application, the pure gluon result can also be used to generate cut results for theories with fewer supersymmetries. This is straightforward for theories that can be viewed as a truncation of $\mathcal{N}=4$ Yang-Mills, i.e., their action can be obtained from the $\mathcal{N}=4$ theory by retaining a subset of fields, forming a $\mathrm{SU}(\mathcal{N})$ representation with $\mathcal{N}<4$, and setting the remaining ones to zero. This corresponds to $\mathcal{N}=1,2$ super-Yang-Mills theories.

To see how to effectively implement the truncation to theories with fewer supersymmetries, we break the $\mathrm{SU}(4)$ R-symmetry group into $\mathrm{SU}(\mathcal{N}) \times \mathrm{SU}(4-\mathcal{N}) \times \mathrm{U}(1)$. The on-shell states of the lower supersymmetric theories transform under the $\mathrm{SU}(\mathcal{N})$ R-symmetry group, but behave as singlets under the remaining $\mathrm{SU}(4-\mathcal{N})$. In terms of index diagrams, the singlet criteria translates to grouping the index lines that carry the $\mathrm{SU}(4-\mathcal{N})$ together, i.e., these index lines must take the same path. This guarantees that the states crossing the cuts are either completely independent of the $\mathrm{SU}(4-\mathcal{N})$ indices, or its dependence is proportional to the $\mathrm{SU}(4-\mathcal{N})$ invariant tensor $\epsilon_{(4-\mathcal{N}) \cdots 4}$.

For an example, consider $\mathcal{N}=2$ super-Yang-Mills theory. We take the contributing index diagrams to be those where index lines labeled by 3 and 4 follow the same path. For external gluons, this gives the following numerator in any MHV cut contributions,

$$
(A+B+C+\ldots)^{2}\left(A^{2}+B^{2}+C^{2}+\ldots\right),
$$

where $A, B, C$ represent the same terms as in eq. (52), and the squares $A^{2}, B^{2}, C^{2}$ are a consequence of the requirement that two indices are always grouped together in the diagrams.

A straightforward generalization to any number of supersymmetries then gives [58]

$$
(A+B+C+\ldots)^{\mathcal{N}} \times\left(A^{4-\mathcal{N}}+B^{4-\mathcal{N}}+C^{4-\mathcal{N}}+\ldots\right), \quad \mathcal{N}<4
$$

which holds for $\mathcal{N}=0,1,2,3$. (The $\mathcal{N}=3$ case is identical to the $\mathcal{N}=4$ super-YangMills case eq. (52), as expected from the well known equivalence of these theories [77].) In eq. (55), the first factor corresponds the supersymmetric summation over index lines with $\mathcal{N}$ independent R-symmetry indices, and the second factor corresponds to the truncation of index diagrams, where $4-\mathcal{N}$ indices are always grouped together. 


\section{Six-dimensional $\mathcal{N}=(1,1)$ on-shell superspace}

In many cases, it is necessary to work in higher dimensions, either for regularization purposes or to study properties of theories in $D>4$ dimensions. As mentioned above, because the $\mathcal{N}=4$ super-Yang-Mills amplitudes are infrared divergent, either dimensional or a massive regularization is needed. At one-loop a theorem guarantees that four-dimensional cuts naively extended to dimensional regularization will capture all contributions. However, no such theorem yet exists at higher loops. To ensure that no terms are dropped even in four dimensions, one should compute the cuts in $D>4$ dimensions or with masses. A simple way to generate massive amplitudes is to again work in higher dimensions, but treat the extra-dimensional momenta as masses which are not integrated over. Of course, one would also like to use a helicity formalism in higher dimensions. Fortunately, as noted earlier, a spinor-helicity formalism does exist in six dimensions [42], along with an on-shell maximal superspace [44]. (For a general discussion of on-shell superspaces see ref. [43].) Here we will describe the six-dimensional helicity formalism, focusing on its application to the construction of loop amplitudes in a manner manifestly consistent with unitarity [12, 59].

\subsection{Tree-level superamplitudes}

In six dimensions where the Lorentz group is $\mathrm{SO}(5,1) \sim \mathrm{SU}^{*}(4)$ a vector is in the antisymmetric representation of $\mathrm{SU}^{*}(4), p^{A B}=-p^{A B}$, where $A, B=1, \cdots, 4$. A null vector can then be written as a spinor product in the bispinor notation as

$$
p^{2}=0 \rightarrow p^{A B}=\lambda^{A a} \lambda_{a}^{B},
$$

where $\lambda^{A a}$ is a six-dimensional chiral spinor, carrying a local SU(2) index, $a$. The momentum $p^{A B}$ is invariant under the local $\mathrm{SU}(2)$ transformation, which gives the correct counting for the degrees of freedom for a massless six-dimensional vector, $4 \times 2-3=5$. However, since the momentum is also invariant under the global part of the $\mathrm{SU}(2)$ transformation, this symmetry is isomorphic to the chiral part of the little group. From now on we will identify the two. A gluon polarization vector can then be readily expressed as [42]

$$
\left.\left.\epsilon_{a \dot{a}}^{\mu}(p, k)=\frac{1}{\sqrt{2}}\left\langle p_{a}\left|\sigma^{\mu}\right| k_{b}\right\rangle\left(\left\langle k_{b}\right| p^{\dot{a}}\right]\right)^{-1}=\left(\left\langle p^{a}\right| k_{\dot{b}}\right]\right)^{-1} \frac{1}{\sqrt{2}}\left[k_{\dot{b}}\left|\tilde{\sigma}^{\mu}\right| p_{\dot{a}}\right],
$$

where $k$ is an arbitrary null vector and we used the bra-ket notation for the chiral $\lambda^{A a}$ and anti-chiral spinor $\tilde{\lambda}_{A \dot{a}}$ respectively. Note that the polarization vector carries the full sixdimensional little-group indices $\mathrm{SO}(4) \sim \mathrm{SU}(2) \times \mathrm{SU}(2)$, i.e. the on-shell states of the gauge field are non-chiral. This is an important distinction from the four-dimensional descendant, where the vector polarization states are chiral.

For the maximal supersymmetric theory in six dimensions, an on-shell superspace has been constructed [44] that has many similarities with the four-dimensional

ๆ Here the " $*$ " indicates it is in the pseudoreal representation. 
version [61]. The six-dimensional on-shell states include four vectors, four scalars, and eight fermions. These states can be packaged into a single scalar superfield, with the supercoordinates $\eta_{a}, \tilde{\eta}_{\dot{a}}$ each carrying a little-group index. The expansion of the superfield in terms of component fields takes the form,

$$
\begin{aligned}
\Phi(\eta, \tilde{\eta})= & \phi+\chi^{a} \eta_{a}+\phi^{\prime}(\eta)^{2}+\psi_{\dot{a}} \tilde{\eta}^{\dot{a}}+g^{a}{ }_{\dot{a}} \eta_{a} \tilde{\eta}^{\dot{a}}+\tilde{\psi}_{\dot{a}}(\eta)^{2} \tilde{\eta}^{\dot{a}} \\
& +\phi^{\prime \prime}(\tilde{\eta})^{2}+\tilde{\chi}^{a} \eta_{a}(\tilde{\eta})^{2}+\phi^{\prime \prime \prime}(\eta)^{2}(\tilde{\eta})^{2} .
\end{aligned}
$$

The projection down to four-dimensional states is done by noting that the fourdimensional $\mathrm{U}(1)$ little group emerges as the diagonal $\mathrm{U}(1)$ of the six-dimensional $\mathrm{SU}(2) \times \mathrm{SU}(2)$.

Using six-dimensional spinors, we define the supermomentum as

$$
q^{A}=\lambda^{A a} \eta_{a}, \tilde{q}_{B}=\tilde{\lambda}_{B \dot{b}} \tilde{\eta}^{\dot{b}} .
$$

These variables give fairly compact representations of the tree amplitudes of sixdimensional maximal super-Yang-Mills theory. For example, at four and five points the amplitudes are 44

$$
\begin{aligned}
& \mathcal{A}_{4}^{\text {tree }}(1,2,3,4)=-\frac{i}{s_{12} s_{23}} \delta^{4}\left(\sum_{i=1}^{4} q_{i}^{A}\right) \delta^{4}\left(\sum_{i=1}^{4} \tilde{q}_{i B}\right), \\
& \mathcal{A}_{5}^{\text {tree }}(1,2,3,4,5)=\frac{\delta^{4}\left(\sum_{i=1}^{5} q_{i}^{A}\right) \delta^{4}\left(\sum_{i=1}^{5} \tilde{q}_{i B}\right)}{s_{12} s_{23} s_{34} s_{45} s_{51}}\left\{q_{1}^{A}\left(p_{2} p_{3} p_{4} p_{5}\right)_{A}{ }_{A}^{B} \tilde{q}_{1 B}+\right.\text { cyclic } \\
& \left.+\frac{1}{2}\left[q_{1}^{A} \tilde{\Delta}_{2 A}+q_{3}^{A} \tilde{\Delta}_{4 A}+\left(q_{3}+q_{4}\right)^{A} \tilde{\Delta}_{5 A}+(\text { chiral conjugate })\right]\right\}
\end{aligned}
$$

where $\tilde{\Delta}_{2 A}=\left(p_{2} p_{3} p_{4} p_{5}-p_{2} p_{5} p_{4} p_{3}\right)_{A}{ }_{A} \tilde{q}_{2 B}$ and chiral conjugation indicates that one exchanges the chirality of the spinors present in the function. The fermionic delta functions are given by

$$
\delta^{4}\left(\sum_{i=1}^{n} q_{i}^{A}\right) \equiv \frac{1}{4 !} \epsilon_{B C D E}\left(\sum_{i=1}^{n} q_{i}^{B}\right)\left(\sum_{i=1}^{n} q_{i}^{C}\right)\left(\sum_{i=1}^{n} q_{i}^{D}\right)\left(\sum_{i=1}^{n} q_{i}^{E}\right),
$$

and a similar expression for the chiral conjugate.

\subsection{Super sewing rules}

We now illustrate some key features in the sewing of tree amplitudes utilizing the $N=(1,1)$ on-shell superspace, either for BCFW recursion or for unitarity cuts. In general, $n$-point tree amplitudes take the schematic form,

$$
\begin{aligned}
& \mathcal{A}_{n} \sim\left[\delta\left(\sum q^{A}\right) \delta\left(\sum \tilde{q}_{A}\right)\right]^{4} q^{n-4} \tilde{q}^{n-4} \text { for } n>3, \\
& \mathcal{A}_{3} \sim\left[\delta\left(\sum q^{A}\right) \delta\left(\sum \tilde{q}_{A}\right)\right]^{2}\left(\sum w \cdot \eta\right)\left(\sum \tilde{w} \cdot \tilde{\eta}\right),
\end{aligned}
$$

where the variables $w$ are additional variables introduced due to the special kinematics of a three-point amplitude [42]. We will not need the precise form here, as we only wish to explain the structure. When sewing amplitudes together, we must sum over all 
physical states that are allowed to propagate across the cuts. As in four dimensions, this sum is taken care of by integrating out the $\eta, \tilde{\eta}$ of the lines being cut.

The presence of super-momentum delta functions again indicates that one can algebraically solve the delta-function constraints and substitute the solutions into the remaining functions. This can be further simplified by combining the delta functions such that a factor $\delta^{4}\left(\sum_{\mathcal{E}} q\right) \delta^{4}\left(\sum_{\mathcal{E}} \tilde{q}\right)$, representing the overall supermomentum conservation over the external legs $\mathcal{E}$, is extracted outside of the integral. Each of the remaining delta function pairs is of degree eight, and hence can be used to localize two pairs of $\eta_{l}, \tilde{\eta}_{l} \mathrm{~S}+$ To implement the localization, it is convenient to rewrite the delta functions as

$$
\delta^{4}\left(q_{i}^{A}+q_{j}^{A}+Q^{A}\right)=s_{i j} \delta^{2}\left(\eta_{i a}+\frac{\left\langle i_{a}\right| \not p_{j} Q}{s_{i j}}\right) \delta^{2}\left(\eta_{j a}+\frac{\left\langle j_{a}\right| \not p_{i} Q}{s_{i j}}\right),
$$

where $\eta_{i}$ and $\eta_{j}$ are the $\eta$ s that the delta functions localize.

We thus have a general procedure to evaluate the supersums in six-dimensional unitarity cuts [12]:

- For a cut diagram with $m$ tree amplitudes, choose $m-1$ of them. These will be the amplitudes whose supermomentum delta function will be used to localize the Grassmann integrals. The remaining delta function will become the overall delta function that is pulled outside of the integral.

- Choose 2(m-1) independent loop supermomenta for the delta functions to localize. (One should choose the loop momenta so that the substitution of the solution does not imply iterative loops, for example, one delta function enforcing $\eta_{l_{1}} \rightarrow \eta_{l_{3}}$ while another enforcing $\eta_{l_{3}} \rightarrow \eta_{l_{1}}$.)

- Using eq. (63) we can then easily carry out all Grassmann integrations and substitute the solution into the remaining functions.

For cuts that contain three-point subamplitudes, the supersum is more involved due to the introduction of additional variables because of the special kinematics. However, we can always choose a spanning set of cuts with no three-point tree amplitudes or alternatively group together any three point amplitudes appearing in the cuts with other tree amplitudes, so as to avoid this technicality. We refer to ref. [12] for details on dealing with this case.

\subsection{One-loop four-point example}

We now illustrate the evaluation of supersums by computing the one-loop four-point maximal super-Yang-Mills in six dimensions. Consider again the two-particle cuts in figure 1(a). Using Grassmann integration to sum the internal states we have

$$
\sum_{s_{1}, s_{2}} \mathcal{A}_{4}\left(-l_{1}^{s_{2}}, 1,2, l_{3}^{s_{1}}\right) \mathcal{A}_{4}\left(-l_{3}^{s_{1}}, 3,4, l_{1}^{s_{2}}\right)
$$

\footnotetext{
+ Recall that each $\eta, \tilde{\eta}$ has two components, this gives a total of eight components to be localized by
} the delta function. 


$$
=-\int d^{2} \eta_{l_{1}} d^{2} \eta_{l_{3}} d^{2} \tilde{\eta}_{l_{1}} d^{2} \tilde{\eta}_{k_{3}} \frac{\delta^{4}\left(\sum_{R} q^{A}\right) \delta^{4}\left(\sum_{R} \tilde{q}_{A}\right)}{\left(p-k_{1}\right)^{2} s} \frac{\delta^{4}\left(\sum_{L} q^{B}\right) \delta^{4}\left(\sum_{L} \tilde{q}_{B}\right)}{s\left(p+k_{4}\right)^{2}} .
$$

where the $\sum_{L}$ and $\sum_{R}$ signify sums over the legs on the left and right sides of the cut in figure 1(a). Pulling out the overall supermomentum delta function we arrive at

$$
\frac{\delta^{4}\left(\sum_{i=1}^{4} q_{i}^{A}\right) \delta^{4}\left(\sum_{i=1}^{4} \tilde{q}_{i A}\right)}{\left(p-k_{1}\right)^{2} s} \frac{\left(l_{1} \cdot l_{3}\right)^{2}}{s\left(p+k_{4}\right)^{2}}=-i s t \mathcal{A}_{\text {tree }}(1,2,3,4) \frac{1}{\left(p-k_{1}\right)^{2}\left(p+k_{4}\right)^{2}},
$$

where we used $l_{3}-l_{1}=k_{1}+k_{2}$, and the explicit form of the four-point superamplitude in eq. (60) to obtain the final line. This answer matches the naive continuation of the four-dimensional result (40) to six dimensions. Thus, when extending the fourdimensional expressions either to dimensional regularization or to massively regularized expressions, all terms are properly captured. The situation is not so simple for cases with fewer supersymmetries or with larger numbers of loops and legs. In these cases the six-dimensional formalism can ensure that no contributions are dropped in the regularization or when extending the results to higher dimensions.

\section{Carrying properties from trees to loops}

We now explain how the unitarity method helps us to carry over novel tree-level properties to loop level. In particular, symmetries of tree-level amplitudes can be carried over to loop level if one can show that all unitarity cuts, combined with the cut propagators, transform in a universal covariant fashion. In doing so, one also needs to track the effect of regularization. In this section we will use dual conformal symmetry as an example showing how the unitarity method can be used to carry over tree properties to loop level and to illustrate regularization issues.

A more complex situation arises when the symmetry or property is directly affected by the cut conditions or when the property is not global. This makes it more difficult to prove that the property carries over to loop level. Nevertheless, the unitarity method offers a powerful tool for studying the property at loop level and for formulating conjectures. We shall illustrate this situation using the recently discovered duality between color and kinematics [35, 36, 14].

\subsection{Dual conformal symmetry}

As discussed earlier, dual conformal symmetry is a conformal symmetry in the dual $x$ space, where $x$ is defined in terms of momenta, as given in eq. (30). This can be extended to a dual superconformal symmetry by introducing the fermionic coordinates [23],

$$
\theta_{i}^{\alpha a}-\theta_{i+1}^{\alpha a}=\lambda_{i}^{\alpha} \eta_{i}^{a}
$$

Once the superamplitudes are written in these dual super coordinates, the dual conformal property can be straightforwardly established through the relationship between the conformal boost generator and the translation generator,

$$
K_{\mu}=I P^{\mu} I,
$$


where $I$ is the inversion operator. The inversion operator acts on $x$ and $\theta$ as

$$
I\left[x_{i \alpha \dot{\beta}}\right]=\frac{x_{i \beta \dot{\alpha}}}{x_{i}^{2}}, \quad I\left[\theta_{i}^{\alpha a}\right]=\frac{x_{i}^{\dot{\alpha} \beta}}{x_{i}^{2}} \theta_{i \beta}^{a} .
$$

The inversion properties of the original on-shell variables, $\left(\lambda^{\alpha}, \tilde{\lambda}^{\dot{\alpha}}, \eta^{a}\right)$, can be determined by demanding that inversion preserves the constraints (30) and (66) [23]. For later use we note that $\lambda_{i}$ inverts as $*$

$$
I\left[\lambda_{i}^{\alpha}\right]=\frac{x_{i}^{\dot{\alpha} \beta} \lambda_{i \beta}}{\sqrt{x_{i}^{2} x_{i+1}^{2}}}=\frac{x_{i+1}^{\dot{\alpha} \beta} \lambda_{i \beta}}{\sqrt{x_{i}^{2} x_{i+1}^{2}}} .
$$

Since our focus will be on loop amplitudes, where loop momenta do not follow cyclic orderings and we cannot alway label the dual variables consecutively, we use the notation

$$
x_{i}-x_{j}=p_{\{i j\}}, \quad \theta_{i}-\theta_{j}=q_{\{i j\}} .
$$

Ref. [23] proved, using super-BCFW recursion relations [25, 49], that the $n$ point tree-level amplitude of $\mathcal{N}=4$ super-Yang-Mills transforms covariantly under dual conformal inversion as

$$
I\left[\mathcal{A}_{n}^{\text {tree }}\right]=\left(\prod_{i=1}^{n} x_{i}^{2}\right) \mathcal{A}_{n}^{\text {tree }}
$$

Here we show that it is straightforward to extend this result to a planar loop-level amplitude statement, via the unitarity method [25, 12, 27],

$$
I\left[\mathcal{A}_{n}^{(L)}\right]=\left(\prod_{i=1}^{n} x_{i}^{2}\right) \mathcal{A}_{n}^{(L)} .
$$

At this point $\mathcal{A}_{n}^{(L)}$ is understood as the $L$-loop amplitude prior to integration, defined without a regulator. Below we describe the effect of the regulator. Our discussion will follow the analogous $D=6$ discussion of ref. [27].

To study the inversion properties in the unitarity cuts, it is more convenient to separate the (super)momentum delta functions from the rest of the tree amplitude. For amplitudes with four or more external legs, we have

$$
\mathcal{A}_{n}^{\text {tree }}=\delta^{4}(P) \delta^{8}(Q) f_{n}, \text { with } I\left[f_{n}\right]=\left(\prod_{i=1}^{n} x_{i}^{2}\right) f_{n} .
$$

We now convert the supersum, which are integrations over $\eta \mathrm{s}$, into integrations over $\theta$. We first note that the total number of dual points for a loop amplitude is $F=n+L$, where $L$ is the loop order and $n$ the number of external legs. The supercut is given by

$$
\begin{aligned}
\left.\mathcal{A}_{n}^{L}\right|_{\text {cut }} & =\int \prod_{\{i j\}} d^{4} \eta_{\{i j\}} \times \mathcal{A}_{(1)}^{\text {tree }} \mathcal{A}_{(2)}^{\text {tree }} \mathcal{A}_{(3)}^{\text {tree }} \ldots \mathcal{A}_{(m)}^{\text {tree }} \\
& =\delta^{4}(P) \int \prod_{\{i j\}} d^{4} \eta_{\{i j\}} \times \prod_{\alpha} \delta^{8}\left(Q_{\alpha}\right) f_{\alpha},
\end{aligned}
$$

\footnotetext{
* Our inversion rules correspond to choosing $\kappa_{i}$ of eq.(4.1) of ref. [25] to be $\sqrt{x_{i}^{2} x_{i+1}^{2}}$.
} 
where we assume the cut is part of a spanning set involving only tree amplitudes with four or more external legs. The product over $\{i j\}$ only runs over internal cut lines while the product over $\alpha$ runs over the tree subamplitudes. Next we use the identity,

$$
\prod_{\alpha} \delta^{8}\left(Q_{\alpha}\right)=\int\left[\prod_{k} d^{8} \theta_{k}\right]\left[\prod_{\{r s\}} \delta^{8}\left(\theta_{r}-\theta_{s}-\lambda_{\{r s\}} \eta_{\{r s\}}\right)\right],
$$

where the product over $\{r s\}$ runs over all lines. Since the translation from supermomentum to dual $\theta$ coordinates has an overall shift symmetry, the integration measure $d^{8} \theta_{k}$ is understood to include only $F-1$ of them. To see the equality (74), one notes that on the LHS the delta functions can be used to localize the $8(F-1)$ integrals. Denoting the total number of lines, internal or external, as $P$, there are then $8(P-F+1)$ delta functions left. For planar diagrams, $P=T+F-1$, where $T$ is the number of tree amplitudes in the cut. Thus, one is left with the supermomentum delta functions for each tree amplitude, as claimed.

Replacing $\delta^{8}\left(Q_{\alpha}\right)$ in eq. (74) by eq. (75), we can now integrate the $\eta \mathrm{s}$. Using the super delta functions to replace the $\eta \mathrm{s}$ in $f_{\alpha}$ with $\theta \mathrm{s}$, the remaining $\eta$ dependence then comes solely from the delta functions. The $\eta$ integral then simply gives

$$
\begin{aligned}
\left.\mathcal{A}_{n}^{L}\right|_{\text {cut }}= & \delta^{4}(P) \int \prod_{k} d^{8} \theta_{k} \times \prod_{\alpha} f_{\alpha} \times \prod_{\{i j\}} \delta^{4}\left(\theta_{i j} \lambda_{\{i j\}}\right) \\
& \times \prod_{\{r s\}} \delta^{8}\left(\theta_{r}-\theta_{s}-\lambda_{\{r s\}} \eta_{\{r s\}}\right),
\end{aligned}
$$

where now $\{r s\}$ only runs over the external lines. Pulling out an overall supermomentum delta function, the remaining $8(n-1)$ delta functions in the product over $\{r s\}$ can be used to localize part of the $\theta_{k}$ integration, leaving only the internal dual points. We are finally left with

$$
\left.\mathcal{A}_{n}^{L}\right|_{\text {cut }}=\delta^{4}(P) \delta^{8}(Q) \int\left(\prod_{l_{k}} d^{8} \theta_{l_{k}}\right) \prod_{\{i j\}} \delta^{4}\left(\theta_{i j} \lambda_{\{i j\}}\right) \prod_{\alpha} f_{\alpha},
$$

where the product over $l_{k}$ now runs only over the internal dual point labels. To calculate the inversion weight of a given cut, we follow the weight of each contributing piece:

- For every internal loop label $l_{k}$, the $\theta_{l_{k}}$ measure contributes a factor $\left(x_{l_{k}}^{2}\right)^{4}$.

- Each internal leg $\{i j\}$ contributes $\left(x_{i}^{2} x_{j}^{2}\right)^{-2}$ which comes from $\delta^{4}\left(\theta_{i j} \lambda_{\{i j\}}\right)$.

- Each tree-level subamplitude $f_{\alpha}$ contributes $\prod_{i} x_{i}^{2}$, where $i$ runs over all tree dual labels.

After restoring the cut propagators, which invert as

$$
I\left[\frac{1}{p_{\{i j\}}^{2}}\right]=I\left[\frac{1}{x_{i j}^{2}}\right]=\frac{x_{i}^{2} x_{j}^{2}}{x_{i j}^{2}},
$$

and combining with the other inversion factors, we see that each planar cut inverts with the overall prefactor,

$$
\left(\prod_{i=1}^{n} x_{i}^{2}\right)\left(\prod_{k=1}^{L}\left(x_{l_{k}}^{2}\right)^{4}\right)
$$


where $x_{l_{k}}$ are internal dual points. One can extend this result to cuts involving threepoint subamplitudes, though as noted above this is unnecessary because spanning sets of cuts without these exist.

If we ignore regularization issues and include the four-dimensional loop integration

measure, the measure will provide an extra inversion weight of $\prod_{k}\left(x_{l_{k}}^{2}\right)^{-4}$, which exactly cancels the extra weight of internal dual points coming from the integrand. In any case, the key result is that all cuts have the same inversion weight. Because there is a uniform inversion for all cuts, we conclude that the $L$-loop amplitude prior to integration must invert as given in eq. (71).

As noted earlier, for the discussion to hold after integration we need a regulator, given the presence of infrared divergences. Fortunately, for $\mathcal{N}=4$ super-Yang-Mills, there are natural and easy to implement regulators. One option is to use dimensional regularization and the other a massive regulator [52]. In either case, the simplest way to study the dual conformal properties is to use the six-dimensional helicity and superspace formalism. With the six-dimensional language we then have simple way to prove the dual conformal properties in the regularized $D=4$ theory to all loop orders, using generalized unitarity

As shown in ref. [27], following similar steps as the four-dimensional case outlined above, the six-dimensional integrand for the $L$-loop amplitude inverts in the same fashion as the four-dimensional integrand. To do so we simply need to extend the dual conformal transformations to act also on the extra-dimensional components [12, 26] (or equivalently masses [52]). Inserting back the loop momentum integral and the overall momentum and supermomentum delta functions, the inversion weight differs from eq. (71) by extra powers of the $x_{i}$. These are due to solely the extra components in the loop integration measure and the momentum delta functions.

On the other hand, if we treat the extra dimensional components as masses, then the loop-momentum integration measure factors and momentum delta functions are identical to the four-dimensional case. From the four-dimensional point of view, the momentum of the extra dimensions is to be identified as regulator masses, and hence should not be integrated over. Thus, as shown in ref. [27], the massively regulated amplitude inverts as in eq. (71), and dual conformal symmetry holds in massively regulated planar $\mathcal{N}=4$ amplitudes to all loop orders, as expected [52]. This may contrasted with dimensional regularization, where the symmetry is anomalous because the inversion weights of the internal dual points do not properly cancel.

\subsection{A duality between color and kinematics}

As a second more intricate example, we consider the question of carrying over a newly discovered duality between color and kinematics from tree level to loop level. (For further details on this duality see the chapter in this review from Carrasco and Johansson [14].)

To understand the proposed duality, we first rearrange an $L$-loop amplitude with 


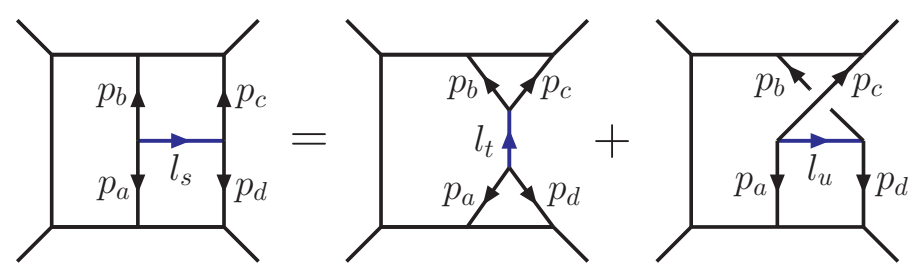

Figure 10. A numerator duality relation at three loops. The relation is either for the color factors or for the diagram numerators.

all particles in the adjoint representation into the form,

$$
(-i)^{L} \mathcal{A}_{n}^{\text {loop }}=\sum_{j} \int \prod_{l=1}^{L} \frac{d^{D} p_{l}}{(2 \pi)^{D}} \frac{1}{S_{j}} \frac{n_{j} c_{j}}{\prod_{\alpha_{j}} p_{\alpha_{j}}^{2}},
$$

where the sum runs over the set of $n$-point $L$-loop diagrams with only cubic vertices. These include distinct permutations of external legs, and the $S_{j}$ are the symmetry factors of each diagram. The form (80) can be obtained straightforwardly, for example, from Feynman diagrams, by representing all contact terms as inverse propagators in the kinematic numerators that cancel propagators. We suppress factors of the coupling constant for convenience. The product in the denominator runs over all propagators of each cubic diagram. The $c_{i}$ are the color factors obtained by dressing every three vertex with an $\tilde{f}^{a b c}=i \sqrt{2} f^{a b c}$ structure constant, and the $n_{i}$ are kinematic numerator factors depending on momenta, polarizations and spinors. For supersymmetric amplitudes expressed in superspace, there will also be Grassmann parameters in the numerators.

According to the color-kinematics duality proposal of refs. [35, 36], arrangements of the diagrammatic numerators in eq. (80) exist such that they satisfy equations in oneto-one correspondence with the color Jacobi identities. That is, for every color Jacobi identity we have a relation between kinematic numerators,

$$
c_{i}=c_{j}-c_{k} \Rightarrow n_{i}=n_{j}-n_{k} .
$$

For example, as illustrated in figure 10, the numerators of the three displayed diagrams satisfy a similar equation as satisfied by the color factors of the diagram.

Perhaps more remarkable than the duality itself, is a related conjecture that once the gauge-theory amplitudes are arranged into a form satisfying the duality (81), corresponding gravity amplitudes can be obtained simply by taking a double copy of gauge-theory numerator factors [35, 36],

$$
(-i)^{L+1} \mathcal{M}_{n}^{\text {loop }}=\sum_{j} \int \prod_{l=1}^{L} \frac{d^{D} p_{l}}{(2 \pi)^{D}} \frac{1}{S_{j}} \frac{n_{j} \tilde{n}_{j}}{\prod_{\alpha_{j}} p_{\alpha_{j}}^{2}},
$$

where the $\tilde{n}_{i}$ represent numerator factors of a second gauge theory amplitude, the sum runs over the same set of diagrams as in eq. (80). We suppressed the gravitational coupling constant in this expression. This is expected to hold in a large class of gravity theories, including theories that are the low-energy limits of string theories. It should 
also hold in pure gravity, but in this case extra projectors would be required to remove the unwanted states arising in the direct product of two pure Yang-Mills theories. At tree level $(L=0)$, this double-copy property is closely related to the KLT relations between gravity and gauge theory [51].

For the tree case, through use of BCFW recursion, the double-copy formula (82) has been proven for pure gravity and for $\mathcal{N}=8$ supergravity tree amplitudes in $D=4$, assuming the duality (81) holds in the corresponding gauge theories [78]. At tree level a consequence of this duality is non-trivial relations between the color-ordered partial amplitudes of gauge theory [35, 79, 80]. The duality has also been studied in string theory [81, 82]. This duality has also been discussed from the Lagrangian vantage point in ref. [78]. Recently, an alternative trace-based representation of the color-kinematics duality was given in [83].

The non-trivial part of the all-loop conjecture (81) is that there is sufficient freedom to arrange gauge-theory multiloop amplitudes in a way that satisfies the color-kinematics duality (81). To analyze whether the conjecture is plausible, we can use the unitarity method.

The present situation is more complicated than the previous example of dual conformal symmetry. In the dual conformal case, when intermediate momenta are placed on shell, the properties are essentially unchanged. This allows the dual conformal properties to easily be carried to loop level, since they hold globally for all cuts. In the present case, when a line is cut, we lose the Jacobi-like relation involving that line, altering the system of equations enforcing the duality. In addition, the remaining numerator relations become simpler because of the extra on-shell cut conditions. The duality (81) is implemented as a collection of identities which act locally on diagrams, but which have global consistency implications. The cuts do not need to satisfy the complete set of duality relations satisfied by the loop amplitude, but only a subset. This makes proving that the color-kinematics duality holds for loop amplitudes more difficult than proving that dual conformal covariance holds in the planar case. Indeed, we still do not have a proof that the color-kinematics duality holds to all loop orders. Whether it does depends on the balance between the number of constraints and the freedom to rearrange the amplitudes as the loop order increases. Nevertheless, the fact that the duality (81) holds for all cuts decomposing loop amplitudes to sums of products of tree amplitudes, assuming it holds at tree level, is a key motivation for extending it to all loop orders [35, 36.

Of course, given that we do not yet have a proof that the color-kinematics duality holds, it is important to check it in explicit examples. The unitarity method has been used to provide such examples. The one- and two-loop four-point amplitudes of $\mathcal{N}=4$ super-Yang-Mills theory and $\mathcal{N}=8$ supergravity, as obtained in ref. [50], are easily shown to satisfy the conjecture (81). Another example is the two-loop four-point identical-helicity amplitude of pure Yang-Mills theory [3], which also has been shown to satisfy the duality [35, 36]. Finally, a rather nontrivial example is the three-loop four-point amplitude of $\mathcal{N}=4$ super-Yang-Mills theory [36]. This example is discussed 
further in another chapter of this review [14], including a discussion of how the colorkinematics duality can be exploited to simplify calculations of the generalized cuts.

\section{Concluding Comments}

In summary, generalized unitarity is an established tool for carrying out loop calculations in gauge and gravity theories, having led to a variety of new nontrivial results. Here we summarized four-dimensional and six-dimensional versions, making use of helicity and on-shell superspaces. The six-dimensional version offers a means of regularizing amplitudes, while retaining key advantages of the four-dimensional version. The unitarity method also offers a straightforward means for carrying any identified tree-level property to loop level. As particular examples, we discussed dual conformal symmetry as well as a new duality between color and kinematics. We expect that in the coming years, generalized unitarity will continue to play a leading role in new calculations and for uncovering exciting new properties of scattering amplitudes.

\section{Acknowledgments}

We are grateful to John Joseph Carrasco, Scott Davies, Tristan Dennen, Lance Dixon, Fernando Febres Cordero, Darren Forde, David Kosower, Harald Ita, Henrik Johansson and Radu Roiban for related collaborations and for many stimulating discussions on the topics described here.

\section{References}

[1] Z. Bern, L. J. Dixon, D. C. Dunbar and D. A. Kosower, Nucl. Phys. B 425, 217 (1994) hep-ph/9403226; Nucl. Phys. B 435, 59 (1995) hep-ph/9409265.

[2] Z. Bern and A. G. Morgan, Nucl. Phys. B 467, 479 (1996) (hep-ph/9511336]; Z. Bern, L. J. Dixon and D. A. Kosower, Ann. Rev. Nucl. Part. Sci. 46, 109 (1996) [hep-ph/9602280].

[3] Z. Bern, L. J. Dixon and D. A. Kosower, JHEP 0001, 027 (2000) hep-ph/0001001].

[4] Z. Bern, L. J. Dixon and D. A. Kosower, Nucl. Phys. B 513, 3 (1998) hep-ph/9708239.

[5] Z. Bern, V. Del Duca, L. J. Dixon and D. A. Kosower, Phys. Rev. D 71, 045006 (2005) hep-th/0410224.

[6] Z. Bern, L. J. Dixon and D. A. Kosower, JHEP 0408, 012 (2004) hep-ph/0404293.

[7] R. Britto, F. Cachazo and B. Feng, Nucl. Phys. B 725, 275 (2005) hep-th/0412103; E. I. Buchbinder and F. Cachazo, JHEP 0511, 036 (2005) hep-th/0506126].

[8] Z. Bern, J. J. M. Carrasco, H. Johansson and D. A. Kosower, Phys. Rev. D 76, 125020 (2007) [0705.1864 [hep-th]].

[9] C. Anastasiou, R. Britto, B. Feng, Z. Kunszt and P. Mastrolia, Phys. Lett. B 645, 213 (2007) hep-ph/0609191; R. Britto and B. Feng, JHEP 0802, 095 (2008) [0711.4284 [hep-ph]]; R. Britto and B. Feng, Phys. Rev. D 75, 105006 (2007) hep-ph/0612089; G. Ossola, C. G. Papadopoulos and R. Pittau, Nucl. Phys. B 763, 147 (2007) hep-ph/0609007]; R. Britto, B. Feng and P. Mastrolia, Phys. Rev. D 78, 025031 (2008) [0803.1989 [hep-ph]]; D. Forde, Phys. Rev. D 75, 125019 (2007) [0704.1835 [hep-ph]]; S. D. Badger, JHEP 0901, 049 (2009) [0806.4600 [hep$\mathrm{ph}]$. 
[10] Z. Bern, J. J. M. Carrasco, L. J. Dixon, H. Johansson and R. Roiban, Phys. Rev. D 78, 105019 (2008) [0808.4112 [hep-th]].

[11] Z. Bern, J. J. M. Carrasco, L. J. Dixon, H. Johansson and R. Roiban, 1008.3327 [hep-th].

[12] Z. Bern, J. J. Carrasco, T. Dennen, Y. t. Huang and H. Ita, 1010.0494 [hep-th], to appear in Phys. Rev. D.

[13] R. Britto, to appear in "Scattering Amplitudes in Gauge Theories", special issue of Journal of Physics A, R. Roiban(ed), M. Spradlin(ed), A. Volovich(ed), 1012.4493 [hep-th].

[14] J. J. M. Carrasco and Henrik Johansson, to appear in "Scattering Amplitudes in Gauge Theories", special issue of Journal of Physics A, R. Roiban(ed), M. Spradlin(ed), A. Volovich(ed).

[15] H. Ita, to appear in "Scattering Amplitudes in Gauge Theories", special issue of Journal of Physics A, R. Roiban(ed), M. Spradlin(ed), A. Volovich(ed).

[16] C. F. Berger et al., [BlackHat Collaboration] Phys. Rev. Lett. 102, 222001 (2009) [0902.2760 [hepph]]; R. K. Ellis, K. Melnikov and G. Zanderighi, Phys. Rev. D 80, 094002 (2009) [0906.1445 [hep-ph]]; C. F. Berger et al. [BlackHat Collaboration], Phys. Rev. D 80, 074036 (2009) [0907.1984 [hep-ph]]; G. Bevilacqua, M. Czakon, C. G. Papadopoulos, R. Pittau and M. Worek, JHEP 0909, 109 (2009). [0907.4723 [hep-ph]]; G. Bevilacqua, M. Czakon, C. G. Papadopoulos et al., Phys. Rev. Lett. 104, 162002 (2010). [1002.4009 [hep-ph]].

[17] C. F. Berger, et al. [BlackHat Collaboration], [1009.2338 [hep-ph]].

[18] C. Anastasiou, Z. Bern, L. J. Dixon and D. A. Kosower, Phys. Rev. Lett. 91, 251602 (2003) hep-th/0309040; Z. Bern, L. J. Dixon and V. A. Smirnov, Phys. Rev. D 72, 085001 (2005) hep-th/0505205.

[19] J. M. Maldacena, Adv. Theor. Math. Phys. 2, 231 (1998) [Int. J. Theor. Phys. 38, 1113 (1999)] hep-th/9711200; S. S. Gubser, I. R. Klebanov and A. M. Polyakov, Phys. Lett. B 428, 105 (1998) [hep-th/9802109]; O. Aharony, S. S. Gubser, J. M. Maldacena, H. Ooguri and Y. Oz, Phys. Rept. 323, 183 (2000) hep-th/9905111.

[20] J. M. Drummond, J. Henn, V. A. Smirnov and E. Sokatchev, JHEP 0701, 064 (2007) hep-th/0607160; J. M. Drummond, G. P. Korchemsky and E. Sokatchev, Nucl. Phys. B 795, 385 (2008) [0707.0243 [hep-th]]; A. Brandhuber, P. Heslop and G. Travaglini, Nucl. Phys. B 794, 231 (2008) [0707.1153 [hep-th]].

[21] J. M. Drummond, J. Henn, G. P. Korchemsky and E. Sokatchev, 0808.0491 [hep-th].

[22] Z. Bern, M. Czakon, L. J. Dixon, D. A. Kosower and V. A. Smirnov, Phys. Rev. D 75, 085010 (2007) hep-th/0610248.

[23] J. M. Drummond, J. Henn, G. P. Korchemsky and E. Sokatchev, Nucl. Phys. B 828, 317 (2010) [0807.1095 [hep-th]].

[24] A. Brandhuber, P. Heslop and G. Travaglini, JHEP 0910, 063 (2009) [0906.3552 [hep-th]]; N. Beisert, J. Henn, T. McLoughlin and J. Plefka, JHEP 1004, 085 (2010) [1002.1733 [hepth]]; G. P. Korchemsky and E. Sokatchev, Nucl. Phys. B 839, 377 (2010) [1002.4625 [hep-th]].

[25] A. Brandhuber, P. Heslop and G. Travaglini, Phys. Rev. D 78, 125005 (2008) [0807.4097 [hep-th]].

[26] S. Caron-Huot and D. O'Connell, 1010.5487 [hep-th].

[27] T. Dennen and Y. t. Huang, 1010.5874 [hep-th].

[28] J. Drummond, to appear in "Scattering Amplitudes in Gauge Theories", special issue of Journal of Physics A, R. Roiban(ed), M. Spradlin(ed), A. Volovich(ed).

[29] J. Henn, to appear in "Scattering Amplitudes in Gauge Theories", special issue of Journal of Physics A, R. Roiban(ed), M. Spradlin(ed), A. Volovich(ed), 1103.1016 [hep-th].

[30] Z. Bern, J. J. Carrasco, L. J. Dixon, H. Johansson, D. A. Kosower and R. Roiban, Phys. Rev. Lett. 98, 161303 (2007) hep-th/0702112.

[31] Z. Bern, J. J. Carrasco, L. J. Dixon, H. Johansson and R. Roiban, Phys. Rev. Lett. 103, 081301 (2009) [0905.2326 [hep-th]].

[32] Z. Bern, J. J. M. Carrasco and H. Johansson, 0902.3765 [hep-th]; H. Nicolai, Physics, 2, 70, (2009); R. P. Woodard, Rept. Prog. Phys. 72, 126002 (2009) 0907.4238 [gr-qc]; L. J. Dixon, 1005.2703 [hep-th]. 
[33] G. Bossard, P. S. Howe and K. S. Stelle, Gen. Rel. Grav. 41, 919 (2009) [0901.4661 [hep-th]]; J. Bjornsson and M. B. Green, JHEP 1008, 132 (2010) [1004.2692 [hep-th]]; G. Bossard, P. S. Howe and K. S. Stelle, 1009.0743 [hep-th]; H. Elvang, D. Z. Freedman and M. Kiermaier, 1003.5018 [hep-th]; R. Kallosh and P. Ramond, 1006.4684 [hep-th]; N. Beisert, H. Elvang, D. Z. Freedman, M. Kiermaier, A. Morales and S. Stieberger, Phys. Lett. B 694, 265 (2010) [1009.1643 [hep-th]].

[34] H. Elvang, D. Z. Freedman and M. Kiermaier, to appear in "Scattering Amplitudes in Gauge Theories", special issue of Journal of Physics A, R. Roiban(ed), M. Spradlin(ed), A. Volovich(ed), 1012.3401 [hep-th].

[35] Z. Bern, J. J. M. Carrasco and H. Johansson, Phys. Rev. D 78, 085011 (2008) [0805.3993 [hep-ph]].

[36] Z. Bern, J. J. M. Carrasco and H. Johansson, Phys. Rev. Lett. 105 (2010) 061602 [1004.0476 [hep-th]].

[37] N. Arkani-Hamed, J. L. Bourjaily, F. Cachazo, S. Caron-Huot and J. Trnka, 1008.2958 [hep-th].

[38] N. Arkani-Hamed, J. L. Bourjaily, F. Cachazo and J. Trnka, 1012.6032 [hep-th].

[39] R. J. Eden, P. V. Landshoff, D. I. Olive, J. C. Polkinghorne, The Analytic S Matrix (Cambridge University Press, 1966).

[40] M. H. Goroff and A. Sagnotti, Phys. Lett. B 160, 81 (1985); Nucl. Phys. B 266, 709 (1986); E. Witten, Commun. Math. Phys. 252, 189 (2004) [hep-th/0312171].

[41] Z. Bern, L. J. Dixon and D. A. Kosower, Phys. Rev. D 71, 105013 (2005) hep-th/0501240; Phys. Rev. D 72, 125003 (2005) hep-ph/0505055; Phys. Rev. D 73, 065013 (2006) hep-ph/0507005; D. Forde and D. A. Kosower, Phys. Rev. D 73, 065007 (2006) hep-th/0507292 ; Phys. Rev. D 73, 061701 (2006) hep-ph/0509358; C. F. Berger, Z. Bern, L. J. Dixon, D. Forde and D. A. Kosower, Phys. Rev. D 75, 016006 (2007) hep-ph/0607014.

[42] C. Cheung and D. O'Connell, JHEP 0907, 075 (2009) [0902.0981 [hep-th]].

[43] R. Boels, JHEP 1001, 010 (2010) arXiv:0908.0738 [hep-th]].

[44] T. Dennen, Y. t. Huang and W. Siegel, JHEP 1004, 127 (2010) [0910.2688 [hep-th]].

[45] M. R. Douglas, 1012.2880 [hep-th]; N. Lambert, C. Papageorgakis and M. Schmidt-Sommerfeld, JHEP 1101, 083 (2011) [1012.2882 [hep-th]].

[46] F. Cachazo and D. Skinner, 0801.4574 [hep-th]; F. Cachazo, 0803.1988 [hep-th].

[47] E. W. Nigel Glover and C. Williams, JHEP 0812, 067 (2008) [0810.2964 [hep-th]]. I Bierenbaum, S. Catani, P. Draggiotis and G. Rodrigo, 1007.0194 [hep-ph]; I. S. Caron-Huot, 1007.3224 [hep$\mathrm{ph}]$.

[48] Z. Bern, J. S. Rozowsky and B. Yan, Phys. Lett. B 401, 273 (1997) hep-ph/9702424.

[49] N. Arkani-Hamed, F. Cachazo and J. Kaplan, 0808.1446 [hep-th].

[50] Z. Bern, L. J. Dixon, D. C. Dunbar, M. Perelstein and J. S. Rozowsky, Nucl. Phys. B 530, 401 (1998) hep-th/9802162.

[51] H. Kawai, D. C. Lewellen and S. H. H. Tye, Nucl. Phys. B 269, 1 (1986); Z. Bern and A. K. Grant, Phys. Lett. B 457, 23 (1999) hep-th/9904026]; Z. Bern, Living Rev. Rel. 5, 5 (2002) gr-qc/0206071.

[52] L. F. Alday, J. M. Henn, J. Plefka and T. Schuster, JHEP 1001, 077 (2010) [0908.0684 [hep-th]]; J. M. Henn, S. G. Naculich, H. J. Schnitzer and M. Spradlin, JHEP 1004, 038 (2010) [1001.1358 [hep-th]]; JHEP 1008, 002 (2010) [1004.5381 [hep-th]].

[53] Z. Bern, L. J. Dixon, D. A. Kosower, R. Roiban, M. Spradlin, C. Vergu and A. Volovich, Phys. Rev. D 78, 045007 (2008) [0803.1465 [hep-th]].

[54] D. A. Kosower, R. Roiban, C. Vergu, [1009.1376 [hep-th]].

[55] F. A. Berends, R. Kleiss, P. De Causmaecker, R. Gastmans and T. T. Wu, Phys. Lett. B 103, 124 (1981); P. De Causmaecker, R. Gastmans, W. Troost and T. T. Wu, Nucl. Phys. B 206, 53 (1982); Z. Xu, D. H. Zhang and L. Chang, TUTP-84/3-TSINGHUA; R. Kleiss and W. J. Stirling, Nucl. Phys. B 262, 235 (1985); J. F. Gunion and Z. Kunszt, Phys. Lett. B 161, 333 (1985); Z. Xu, D. H. Zhang and L. Chang, Nucl. Phys. B 291, 392 (1987).

[56] M. Bianchi, H. Elvang and D. Z. Freedman, JHEP 0809, 063 (2008) [0805.0757 [hep-th]]; 
M. Kiermaier, H. Elvang and D. Z. Freedman, JHEP 0906, 068 (2009) [0811.3624 [hep-th]];

M. Kiermaier and S. G. Naculich, JHEP 0905, 072 (2009) [0903.0377 [hep-th]].

[57] H. Elvang, D. Z. Freedman and M. Kiermaier, JHEP 0904, 009 (2009) [0808.1720 [hep-th]].

[58] Z. Bern, J. J. M. Carrasco, H. Ita, H. Johansson and R. Roiban, Phys. Rev. D 80, 065029 (2009) [0903.5348 [hep-th]].

[59] A. Brandhuber, D. Korres, D. Koschade and G. Travaglini, 1010.1515 [hep-th].

[60] A. Brandhuber, W. Spence, G. Travaglini, to appear in "Scattering Amplitudes in Gauge Theories", special issue of Journal of Physics A, R. Roiban(ed), M. Spradlin(ed), A. Volovich(ed).

[61] V. P. Nair, Phys. Lett. B 214, 215 (1988).

[62] M. B. Green, J. H. Schwarz and L. Brink, Nucl. Phys. B 198, 474 (1982).

[63] M. T. Grisaru, H. N. Pendleton and P. van Nieuwenhuizen, Phys. Rev. D 15, 996 (1977); M. T. Grisaru and H. N. Pendleton, Nucl. Phys. B 124, 81 (1977); H. Elvang, D. Z. Freedman and M. Kiermaier, 0911.3169 [hep-th].

[64] M. L. Mangano and S. J. Parke, Phys. Rept. 200, 301 (1991); L. J. Dixon, in QCD \& Beyond: Proceedings of TASI '95, ed. D. E. Soper (World Scientific, 1996) hep-ph/9601359.

[65] L. Dixon, to appear in "Scattering Amplitudes in Gauge Theories", special issue of Journal of Physics A, R. Roiban(ed), M. Spradlin(ed), A. Volovich(ed).

[66] A. Ferber, Nucl. Phys. B 132, 55 (1978).

[67] L. J. Dixon, J. M. Henn, J. Plefka and T. Schuster, 1010.3991 [hep-ph].

[68] S. Lal and S. Raju, Phys. Rev. D 81, 105002 (2010) [0910.0930 [hep-th]], H. Elvang, Y. t. Huang and C. Peng, 1102.4843 [hep-th].

[69] F. Cachazo, P. Svrcek and E. Witten, JHEP 0409, 006 (2004) hep-th/0403047.

[70] R. Britto, F. Cachazo, B. Feng and E. Witten, Phys. Rev. Lett. 94, 181602 (2005) hep-th/0501052.

[71] G. Georgiou, E. W. N. Glover and V. V. Khoze, JHEP 0407, 048 (2004) hep-th/0407027; Y.t. Huang, Phys. Lett. B 631, 177 (2005) hep-th/0507117; H. Feng and Y.-t. Huang, JHEP 0904, 047 (2009) hep-th/0611164.

[72] J. M. Drummond and J. M. Henn, JHEP 0904, 018 (2009) [0808.2475 [hep-th]].

[73] D. A. Kosower, Phys. Rev. D 71, 045007 (2005) hep-th/0406175]; I. Bena, Z. Bern and D. A. Kosower, Phys. Rev. D 71, 045008 (2005) hep-th/0406133.

[74] J. M. Drummond, J. M. Henn and J. Plefka, JHEP 0905, 046 (2009) arXiv:0902.2987 [hep-th]].

[75] N. Beisert, to appear in "Scattering Amplitudes in Gauge Theories", special issue of Journal of Physics A, R. Roiban(ed), M. Spradlin(ed), A. Volovich(ed).

[76] S. Mandelstam, Nucl. Phys. B 213, 149 (1983); P. S. Howe, K. S. Stelle and P. K. Townsend, Nucl. Phys. B 214, 519 (1983); L. Brink, O. Lindgren and B. E. W. Nilsson, Phys. Lett. B 123, 323 (1983).

[77] W. Nahm, Nucl. Phys. B 135, 149 (1978); E. Witten, Phys. Lett. B 77, 394 (1978); A. Galperin, E. Ivanov, S. Kalitsyn, V. Ogievetsky and E. Sokatchev, Phys. Lett. B 151, 215 (1985).

[78] Z. Bern, T. Dennen, Y. t. Huang and M. Kiermaier, 1004.0693 [hep-th].

[79] N. E. J. Bjerrum-Bohr, P. H. Damgaard and P. Vanhove, Phys. Rev. Lett. 103, 161602 (2009) [0907.1425 [hep-th]]; S. Stieberger, 0907.2211 [hep-th].

[80] B. Feng, R. Huang and Y. Jia, 1004.3417 [hep-th].

[81] N. E. J. Bjerrum-Bohr, P. H. Damgaard, T. Sondergaard and P. Vanhove, 1003.2403 [hep-th]; C. R. Mafra, 1007.3639 [hep-th].

[82] S. H. Henry Tye and Y. Zhang, JHEP 1006, 071 (2010) [1003.1732 [hep-th]].

[83] Z. Bern and T. Dennen, 1103.0312 [hep-th]. 